\title{
A Systematic Review on Materno-Foetal Outcomes in Pregnant Women with IgA Nephropathy: A Case of "Late-Maternal" Preeclampsia?
}

\author{
Giorgina Barbara Piccoli ${ }^{1,2, *(1)}$, Isabelle Annemijn Kooij ${ }^{3}$, Rossella Attini ${ }^{3}$, \\ Benedetta Montersino ${ }^{3}$, Federica Fassio ${ }^{3}{ }^{(1)}$, Martina Gerbino ${ }^{3}$, Marilisa Biolcati ${ }^{3}$, \\ Gianfranca Cabiddu ${ }^{4}$ (i), Elisabetta Versino ${ }^{5}$ and Tullia Todros ${ }^{3}$ \\ 1 Dipartimento di Scienze Cliniche e Biologiche, Università di Torino, 10100 Torino, Italy \\ 2 Nephrologie, Centre Hospitalier Le Mans, Avenue Roubillard, 72000 Le Mans, France \\ 3 Unità Materno-Fetale, Dipartimento di Chirurgia, Università di Torino, 10100 Torino, Italy; \\ isabelle.kooij@hotmail.com (I.A.K.); rossella.attini@gmail.com (R.A.); benedettamontersino@yahoo.it (B.M.); \\ federica.fassio@hotmail.it (F.F.); martigerby@hotmail.it (M.G.); marilisa.biolcati@unito.it (M.B.); \\ tullia.todros@unito.it (T.T.) \\ 4 Nefrologia, Ospedale Brotzu, 09100 Cagliari, Italy; gianfranca.cabiddu@tin.it \\ 5 Epidemiologia, Dipartimento di Scienze Cliniche e Biologiche, Università di Torino, 10100 Torino, Italy; \\ elisabetta.versino@unito.it \\ * Correspondence: gbpiccoli@yahoo.it; Tel.: +33-669-733-371
}

Received: 1 July 2018; Accepted: 10 August 2018; Published: 11 August 2018

Abstract: Background: IgA nephropathy is the most common primary glomerulonephritis in pregnancy and shares with other immunologic diseases and kidney diseases a relationship with adverse maternal outcomes, whose entity and pattern is only partially quantified. Recent studies provide new information and a systematic review regarded progression of kidney disease. The discussion of the outcomes with respect to low-risk pregnancies may help to perfect the estimation of the risks, and to identify specific research needs. Methods: A search strategy was built on Medline, EMBASE and the Cochrane review for the period January 2000-April 2017, aimed at retrieving both case series (defined as with at least 6 pregnancies in women with IgA nephropathy) and case reports, to look into rare occurrences. All papers, with or without control groups, were selected if they reported on at least one pregnancy outcome, or on long-term kidney function. Search strategy, paper selection and data extraction were done in duplicate (PROSPERO N 42016042623). Meta-analysis of case series was performed with Metanalyst Beta 3.13. Case reports were analysed narratively. Results: The search retrieved 556 papers, of which 27 were included (13 series and 14 case-reports). The case series report on 581 women with 729 pregnancies. The analysis was performed in comparison to the available control groups: 562 non-pregnant controls were available for the analysis of progression of kidney disease. As for pregnancy related outcomes (preeclampsia (PE), pregnancy induced hypertension $(\mathrm{PIH})$, preterm birth, small babies), we meta-analyzed the data with respect to the only series of low-risk pregnancies (1418 pregnancies). When compared with women who never got pregnant after diagnosis of IgA nephropathy, in the present meta-analysis pregnancy in women with IgA nephropathy was not associated with a higher risk of progression of kidney disease, possibly due to the overall preserved kidney function at baseline: end-stage kidney disease (OR 0.68; CI 0.28-1.65). Conversely, the incidence of adverse pregnancy-related outcomes was increased compared to low-risk controls: PE and PIH were more than ten-fold increased (OR 11.80; CI 7.53-18.48 and OR 10.39; CI 5.45-19.80), while the increase in risk of preterm birth and "low birth weight babies" was less marked (OR 3.37; CI 1.91-5.95 and OR 2.36; CI 1.52-3.66), a discrepancy suggesting the occurrence of "late" or "maternal" PE, that may affect less severely foetal growth or shorten gestation. In conclusion, in the present meta-analysis IgA nephropathy was not associated with an increased progression of kidney disease. The more than ten-fold increased risk of PIH and PE, in combination with a doubled 
risk of small babies, suggests the occurrence of "late" or "maternal" PE, usually less affecting early foetal growth. This finding may be of help in defining control policies, while further research is needed to guide clinical management.

Keywords: IgA nephropathy; pregnancy; preeclampsia; proteinuria; preterm delivery; systematic review

\section{Introduction}

IgA nephropathy is probably the most common primary glomerular nephritis worldwide; its higher incidence in young people makes it highly relevant in pregnancy [1].

The present term of IgA nephropathy encompasses two previously defined diseases, usually known by their eponyms: Berger's disease, in which the IgA deposition is limited to the kidney, and Henoch-Shönlein, in which IgA nephropathy is a part of a systemic vasculitis that could involve the skin and the gastro-intestinal tract, with asymmetric acute osteoarthritis [2,3]. The recognition of family clustering of the two diseases, and the identical renal pathology led to gather both diseases under a unifying definition [4-6]. IgA nephropathy may present with a wide spectrum of clinical presentations, from mild, recurrent and remittent microhematuria to boosts of macrohematuria and nephritic syndrome, or a severe and rapidly progressive disease $[1,4,7,8]$. Hypertension and proteinuria are considered as negative outcome predictors, even if only mild. Their negative effect on outcome is observed even in the absence of reduced renal function, which is a likewise (albeit tautological) acknowledged progression marker itself. Other histological markers of disease progression and risk are still subject of debate [7-14]. The risk of progression to end stage renal disease (ESRD) has been differently evaluated. However, this risk is not negligible over the entire life span as progression to ERSD is reported from rare to frequent, depending on morphological, clinical and laboratory characteristics, and treatment. For these reasons, IgA nephropathy is usually considered as a disease that may remit, but that is never completely cured [14-18].

The acknowledgement of the importance of early renal disease in pregnancy is particularly relevant for IgA nephropathy, due to its relatively high prevalence in young age, slow progression, with frequent preservation of the kidney function at least for long periods, making normal kidney function common in affected patients in childbearing age, and heterogeneous presentation, that may impair early diagnosis [19-22].

As will be further discussed in detail, a systematic review, encompassing a wide time span, was recently focused on the progression of chronic kidney disease in patients with IgA nephropathy who had been pregnant [23]. To try to further add to the knowledge in this important field, we considered several recent publications, not available at the time of the first review, and we focused a second systematic review on the effect of IgA nephropathy on pregnancy and of pregnancy on the progression of IgA nephropathy, on the new millennium, on the account of the changes in disease management and maternofetal care occurring over time, and we attempted for the first time a meta-analytical comparison with a low-risk population, in order to better quantify the risk of the various adverse pregnancy outcomes, as a further support for counseling.

\section{Methods}

\subsection{Eligibility Criteria}

The eligibility criteria were broad, because of the expected retrieval of heterogeneous designs and definitions. Consequently, we included all published studies that dealt with IgA nephropathy in pregnancy and that reported on at least one pregnancy-related outcome, or on long-term kidney function. We included all study designs, i.e., prospective or retrospective cohort studies, case-control studies, trial-based analyses, and case reports. Reviews, whether or not systematic, were also gathered 
to check for papers eventually escaped from our wide search strategy. The search was not limited to papers which provided a control group, but information on all control groups were gathered.

Studies were divided into those regarding more than 5 or less than 6 cases (defined as case series and cases reports, respectively).

The review was prospectively registered on PROSPERO International prospective register of systematic reviews (N 42016042623).

The review was performed according to the Meta-analysis of observational studies in epidemiology (MOOSE) criteria.

\subsection{Search Strategy}

We searched MEDLINE, PubMed, Embase and the Cochrane Review database from January 2000 to April 30th 2017 using a combination of MeSH terms and keywords related to IgA nephropathy and pregnancy, including: pregnancy, pregnant, gestation* and IgA nephropathy, Henoch* or Shoenlein*, Berger*. We also checked the reference lists of relevant articles and citations of included studies.

We did not limit our search to any language, but we limited it to the period starting from 2000, allowing for the considerable changes in management of the disease and in maternofetal follow-up that occurred in the new millennium, in order to implement the clinical decision rules presently recommended by the very few guidelines or consensus statements dealing with glomerular diseases in pregnancy [24-26].

All articles identified were screened for eligibility on the basis of the content only: all papers reporting on any form of IgA nephropathy in pregnancy and reporting on any outcome were selected. The search was performed by G.B.P. and I.A.K., after which I.A.K. and R.A. did a first screening of the papers. The final selection was agreed between G.B.P. and I.A.K., any discrepancy was resolved by discussion.

\subsection{Data Extraction and Quality Assessment}

Two reviewers (I.A.K. and G.B.P.) extracted study and population characteristics and outcomes. A third reviewer (G.C.) double-checked the accuracy of data entered from each study in case of discrepancies. All definitions of IgA nephropathy, pre-eclampsia (PE), pregnancy induced hypertension $(\mathrm{PIH})$, small babies, small for gestational age babies (SGA) or other pregnancy related outcomes were gathered.

Due to the lack of randomized controlled trials (RCTs), and to the limited number and type of control groups, no formal analysis of risk of bias was performed. No selection for quality was performed. However, the studies were critically analyzed and the completeness of the data was considered as an indirect marker of quality.

The case series were compared with the controls available, regarding progression of chronic kidney disease (CKD), and with the only low risk population available, regarding the various pregnancy outcomes. Odds ratios and confidence intervals were calculated (OR, CI). The case reports were described narratively.

\subsection{Data Synthesis}

The PICOS criteria were adapted to this particular situation in which pregnancy is considered the "intervention" as follows:

P-Patients: women with IgA nephropathy in pregnancy;

I-Intervention: in the absence of a true "intervention", pregnancy was considered as the "intervention";

C-Comparators: in the case of progression of kidney disease, non-pregnant IgA patients were considered as comparators. In the case of pregnancy outcomes, considered low-risk pregnancies or pregnancies in women not affected by IgA nephropathy (or other kidney diseases); 
$\mathrm{O}-$ Outcomes: progression of kidney disease; all the materno-fetal outcomes described in the papers (including, but not limited to, PE, PIH, preterm delivery, small babies, SGA).

S-Studies: all studies, regardless of the study design, reporting on pregnancy outcomes or on long-term kidney function in patients with IgA nephropathy.

Statistical analysis was performed using Metanalyst Beta 3.13.

Due to the high baseline heterogeneity a random effect model was employed.

Stratification for baseline renal function, hypertension and proteinuria was attempted, but was not possible due to the high heterogeneity of the papers.

The control groups retrieved mainly regarded progression of kidney disease in women with IgA nephropathy who did not get pregnant after diagnosis; these data were used to analyze the effect of pregnancy on kidney function.

For pregnancy-related outcomes, we employed as a control group the only low-risk population available (from the cohort named TOCOS from the Torino Cagliari Observational Study) and meta-analyzed each study with respect to it.

\section{Results}

\subsection{Case Series: Overall Data}

The search strategy identified 556 articles, of which 27 met the inclusion criteria: 13 case series, which we attempted to meta-analyze, for the various outcomes, and 14 case reports, discussed narratively (Figure 1) [27-53].

Pregnancy in IgA Nephropathy: case series and case reports

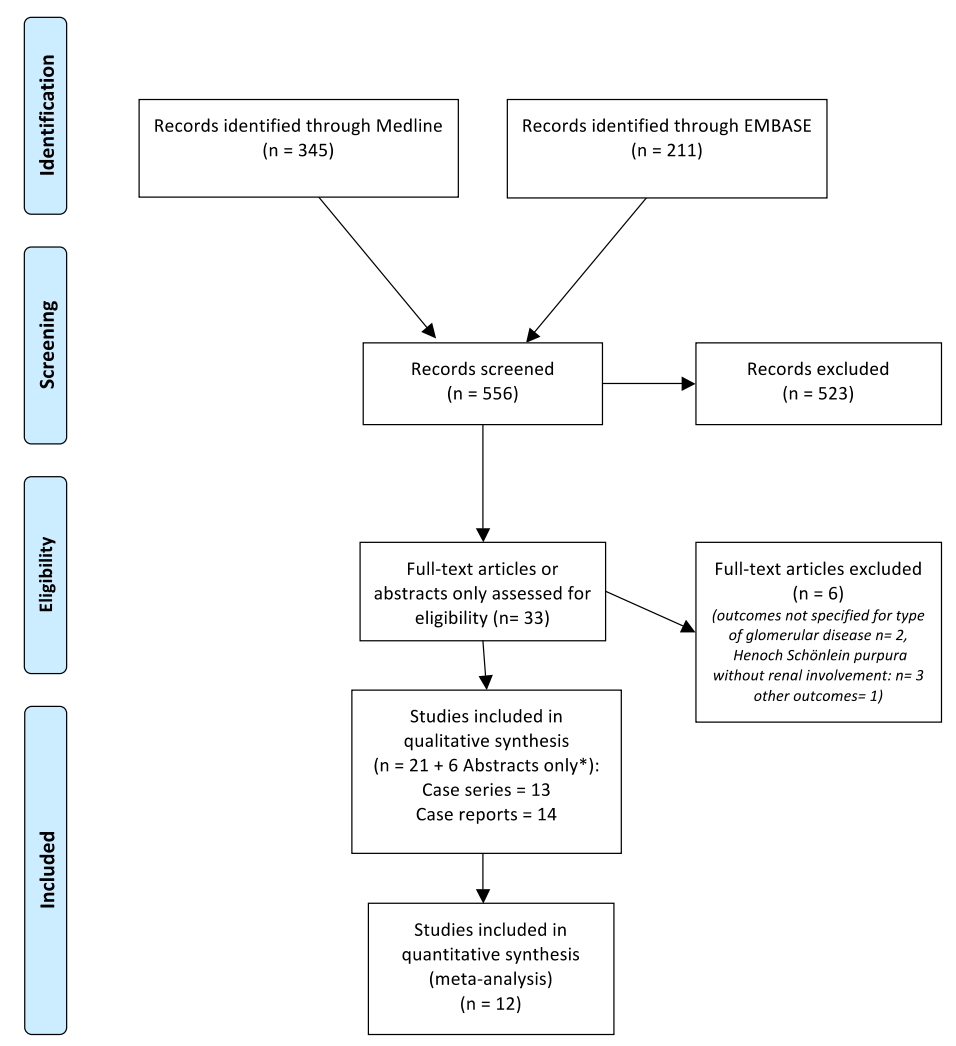

* In 4 cases only the abstract was available (congress proceedings), and in 2 the paper was not retrieved. In all cases the authors and publishers were contacted to obtain further data.

Figure 1. The flow-chart of the retrieved papers. 
The main characteristics of the case series are reported in Tables 1-4 [27-39].

Overall, the case series included four prospective studies, eight retrospective studies and two matched cohort studies, the latter regarding progression of CKD in women affected by IgA nephropathy, with or without pregnancy (Table 1). Globally, the studies report on 581 women with 729 pregnancies in IgA nephropathy (assuming one pregnancy per woman if not otherwise specified), and on 562 non-pregnant controls distributed over three studies; conversely, normal pregnancies were available in only one study [30].

The studies are heterogeneous for the number of pregnancies (12 to 229), setting of study (seven from Asia, five from Europe, one from the USA and one from Saudi Arabia), and study aims. Six studies provided information on the type and frequency of control policies in pregnancy and during follow-up (Table 2).

\subsection{Case Series: Pregnancy Outcomes}

Table 3 reports on the main pregnancy-related outcomes reported in the papers. Given the different study designs, the included studies reported on different outcomes: neonatal deaths were reported on by 4 studies, live births were reported on by 8 studies, preterm delivery was reported by 8 , and need for admission to neonatal intensive care unit by one study only. All studies reported on preeclampsia (PE) and/or hypertensive disorders of pregnancy. Information on birth weight was available in most studies, but the information was not always contextualized to gestational age, and the information on neonates small for gestational age was available in two studies only.

Overall, the papers describe a population with high incidence of PIH (reported in around $30 \%$ of the cases, however, the distinction with preexisting hypertension is usually lacking, and this leads to a possible underestimation of new onset hypertension) and PE (reported in around 15\% of the cases), with about $15 \%$ incidence of preterm deliveries, usually without exhaustive specification of gestational age (Table 3).

Table 4 reports on baseline kidney function and other maternal outcomes: baseline kidney function (glomerular filtration rate: GFR) was relatively well preserved in most studies but was fully normal in 2 only (GFR was $>100 \mathrm{~mL} / \mathrm{min}$ in 2 studies and $<100 \mathrm{~mL} / \mathrm{min}$ in 7 studies). Mean proteinuria was around $1 \mathrm{~g} /$ day. Prevalence of hypertension was not clearly defined at baseline, and the distinction between previous use of angiotensin converting enzyme-inhibitors (ACEi) or Angiotensin II receptor blockers (ARBS) for proteinuria, hypertension or both was not available.

Four of five studies reporting on kidney function over time did not find any difference between cases and non-pregnant controls during follow-up, considering however different outcomes (start of dialysis, doubling of serum creatinine, GFR decrease, new-onset hypertension); the fifth, most recent study, found a correlation between kidney function worsening and the occurrence of adverse pregnancy outcomes [27] (Table 4).

\subsection{Case Reports}

The main characteristics of the case reports are summarized in Tables 5-7 [40-53].

Keeping in mind the aim to discuss severe or unusual situations, the cases reported on various situations, including new onset-diagnosis of IgA nephropathy in pregnancy, recurrence of IgA nephropathy in a kidney allograft, severe reduction of the kidney function (5 cases); one case reported on three pregnancies in the same woman (one occurring in the pre-dialysis phase, two on dialysis) (Table 5).

Presumably as a consequence of the different selection of the cases compared to that of the series, most of the babies were born preterm (61.5\%); maternal complications were common $(30 \%)$ and more than half of the newborns had low birth weight. Nevertheless, all reported babies had a favorable outcome. Conversely, dialysis was needed in pregnancy in three cases and half of the patients developed hypertension or preeclampsia. 
Table 1. IgA nephropathy: Main characteristics of the case series (at least 6 pregnancies).

\begin{tabular}{|c|c|c|c|c|c|}
\hline Author Year [Ref] & Years & Country & Study & Objective, as Stated in the Study & $\begin{array}{l}\text { Pregnancies (P) } \S \S \\
\text { Women (W) }\end{array}$ \\
\hline Park 2017 [27] & 1979-2015 & Korea & Ret & $\begin{array}{l}\text { To assess the relationship between pregnancy and renal prognosis in women with IgAN and to } \\
\text { investigate further whether obstetric complications are associated with renal prognosis }\end{array}$ & $\begin{array}{l}59 \mathrm{~W} \\
64 \mathrm{P} \\
59 \mathrm{~W} \text { controls (non-pregnant IgA) }\end{array}$ \\
\hline O'Shaughnessy 2017 [28] & 1996-2015 & USA & Ret & To investigate the influence of glomerular disease subtype on pregnancy outcomes & $\begin{array}{l}17 \mathrm{~W} \\
18 \mathrm{P}\end{array}$ \\
\hline Su 2017 [29] & 2003-2014 & China & Pro & $\begin{array}{l}\text { To assess the effects of pregnancy on kidney disease progression and risk factors for adverse } \\
\text { pregnancy outcomes in patients with IgAN }\end{array}$ & $\begin{array}{l}104 \mathrm{~W} \\
116 \mathrm{P} \\
309 \mathrm{~W} \text { controls (non-pregnant IgA) }\end{array}$ \\
\hline Tocos 2017 [30] & 2000-2016 & Italy & Pro & To evaluate the maternofetal outcomes in different glomerulonephritis & $\begin{array}{l}27 \mathrm{~W}^{\S} \\
33 \mathrm{P} \\
1418 \mathrm{P} \text { controls (low risk) }\end{array}$ \\
\hline Liu 2014 [31] & 2003-2012 & China & Matched-cohort & $\begin{array}{l}\text { To evaluate the safety of pregnancy in women with IgAN, as well as their risk factors for adverse } \\
\text { pregnancy outcomes, as compared to non-pregnant women with IgAN }\end{array}$ & $\begin{array}{l}62 \mathrm{~W} \\
69 \mathrm{P} \\
62 \mathrm{~W} \text { controls (non-pregnant IgA) }\end{array}$ \\
\hline Oh 2011 [32] & 2004-2009 & Korea & Ret & $\begin{array}{l}\text { To investigate whether higher proteinuria at conception predicts a faster decline in maternal } \\
\text { renal outcomes and to identify whether a proteinuria reduction prior to pregnancy attenuates the } \\
\text { deterioration of postnatal maternal outcomes }\end{array}$ & $52 \mathrm{~W}$ \\
\hline Suetsugu $2011(* *)[33]$ & NR & Japan & Ret & $\begin{array}{l}\text { To explore the clinical characteristics of predictive factors for hypertension in biopsy-proven IgA } \\
\text { nephropathy patients with superimposed preeclampsia }\end{array}$ & $34 \mathrm{~W}$ \\
\hline Shimizu 2010 [34] & 1995-2006 & Japan & Pro & To evaluate the impact of the CKD staging in patients with IgAN on pregnancy and delivery & $\begin{array}{l}29 \mathrm{~W} \\
29 \mathrm{P} \\
45 \mathrm{~W} \text { controls (non-pregnant IgA) }\end{array}$ \\
\hline Waness 2010 [35] & 2000-2006 & $\begin{array}{l}\text { Saudi } \\
\text { Arabia }\end{array}$ & Pro & $\begin{array}{l}\text { To examine the natural history of pregnancies and their impact on renal function in Saudi } \\
\text { females affected by IgAN }\end{array}$ & $\begin{array}{l}12 \mathrm{~W} \\
12 \mathrm{P}\end{array}$ \\
\hline Limardo $2010[36]$ & 1974-2003 & Italy & $\begin{array}{l}\text { Ret multicenter } \\
\text { cohort }\end{array}$ & $\begin{array}{l}\text { To compare the long-term outcome of kidney disease in women with IgAN and preserved } \\
\text { kidney function }(\mathrm{sCr}<1.2 \mathrm{mg} / \mathrm{dL}) \text { who did and did not become pregnant. Data on } 10 \text { pregnant } \\
\text { and } 12 \text { non pregnant women with } \mathrm{s} C \mathrm{r}>1.2 \mathrm{mg} / \mathrm{dL} \text { also gathered }\end{array}$ & $\begin{array}{l}136 \mathrm{~W} \\
229 \mathrm{P} \\
87 \mathrm{~W} \text { controls (non-pregnant IgA) }\end{array}$ \\
\hline Donggyu $2010\left(^{*}\right)[37]$ & 1987-2008 & Korea & Ret & To clarify the influence of pregnancy on the natural course of IgAN & $\begin{array}{l}25 \mathrm{~W} \\
28 \mathrm{P}\end{array}$ \\
\hline Ronkainen 2006 [38] & NR & Finland & Ret & $\begin{array}{l}\text { To evaluate renal survival, morbidity, pregnancy complications and factors predicting outcome } \\
\text { after childhood IgAN }\end{array}$ & $\begin{array}{l}10 \mathrm{~W} \\
22 \mathrm{P}\end{array}$ \\
\hline Ronkainen 2002 [39] & NR & Finland & Ret & $\begin{array}{l}\text { To assess long-term outcome of children with renal involvement at onset of Henoch-Schönlein } \\
\text { purpura by comparison with those who have mainly extra-renal symptoms at referral }\end{array}$ & $\begin{array}{l}14 \mathrm{~W} \\
23 \mathrm{P}\end{array}$ \\
\hline \multicolumn{5}{|c|}{ Overall number of women, pregnancies and controls } & $\begin{array}{l}581 \mathrm{~W} \\
729 \mathrm{P} \S \\
562 \text { non pregnant IgA controls } \\
1418 \text { low risk controls }\end{array}$ \\
\hline
\end{tabular}

CKD: chronic kidney disease; IgAN: IgA nephropathy; NR: not reported; P: pregnancy; Pro: prospective; Ret: retrospective; sCr: serum creatinine; W: woman. ${ }^{*}$ Abstract only (congress proceeding); $\left.{ }^{* *}\right)$ paper in Japanese, abstract used. $\$$ not mentioned in article, additional information available in original database $\$ \S$ if number of pregnancies not specified, we assumed 1 pregnancy per woman. 
Table 2. IgA nephropathy: maternal and fetal control policies, in the papers reporting on them (Case Series).

\begin{tabular}{ll}
\hline Author Year [Ref] & Control Policies \\
\hline Su 2017 [29] & Follow up at least once a month before delivery, and every 1-6 months after delivery, with minimum follow up 12 months postpartum or until dialysis treatment \\
\hline Tocos 2017 [30] & Follow up at least once monthly if proteinuria, hypertension or kidney function reduction \\
\hline Liu 2014 [31] & Follow up every $\leq 1$ month; eGFR decline; determination time-averaged MAP and proteinuria every 3 months \\
\hline Shimizu 2010 [34] & $\begin{array}{l}\text { BP, proteinuria, blood analysis and eGFR at the baseline at the time of detection of pregnancy; at 16, 22 and } 30 \text { weeks of pregnancy; at the time of delivery; and at 3 } \\
\text { months and 1, 2 and 3 years after delivery }\end{array}$ \\
\hline Waness 2010 [35] & Monthly measures of BP, 24 h proteinuria, sCr, CCr; close monitoring and follow up \\
\hline Limardo 2010 [36] & Information gathered at time of biopsy and every 5 year thereafter: CCr, 24 h proteinuria, body weight, BP, therapy with ACEIs/ARBs or immunosuppressants \\
\hline Donggyu 2010 $\left(^{*}\right)[37]$ & sCr followed up max 3 years after delivery \\
\hline
\end{tabular}

ACEI: ACE-inhibitor; ARB: angiotensin II-receptor blocker; BP: blood pressure; CCr: creatinine clearance; eGFR: estimated glomerular filtration rate, assessed by MDRD or EPI formula; IgAN: IgA nephropathy; MAP: mean arterial pressure; NR: not reported; sCr: serum creatinine; * Abstract only.

Table 3. IgA Nephropathy: Fetal and pregnancy outcomes in the case series.

\begin{tabular}{|c|c|c|c|c|c|c|c|c|c|}
\hline \multirow{2}{*}{ Author Year [Ref] } & \multicolumn{5}{|c|}{ All Cases Considered } & \multicolumn{2}{|l|}{ Live Births Only } & \multirow{2}{*}{$\begin{array}{l}\text { All Cases } \\
\text { PE-HT }\end{array}$} & \multirow{2}{*}{$\begin{array}{l}\text { All Cases, or As Stated } \\
\text { Other }\end{array}$} \\
\hline & $\mathbf{P}$ & $\begin{array}{l}\text { Abort. } \\
\text { Spont. } \\
\text { Induced }\end{array}$ & Still Birth & Live Birth & Neo. Death & $\begin{array}{l}\text { Preterm }<37 \text { weeks } \\
\text { Early }<34 \text { weeks } \\
\text { Extreme }<28 \text { weeks }\end{array}$ & NICU & & \\
\hline Park 2017 [27] & 64 & $\mathrm{NR}$ & NR & NR & NR & $\begin{array}{l}\text { Preterm: } 21(33 \%) \\
\text { Early: } 8(13 \%)\end{array}$ & NR & PE: $13(20 \%)$ & $\begin{array}{l}\text { LBW <2500 g: } 16(25 \%) \\
\text { LBW <1500 g: } 6(9 \%) \\
\text { SGA <10th: } 6(9 \%)\end{array}$ \\
\hline $\begin{array}{l}\text { O'Shaughnessy } 2017 \\
\text { [28] }\end{array}$ & 18 & NA & 0 & $18(100 \%)$ & $2(11 \%)$ & $\begin{array}{l}\text { Preterm <37 w: } 6(33 \%), 5 / 6 \\
\text { induced/CS on maternal } \\
\text { indication } \\
\text { Preterm <32 w: } 4(22 \%)\end{array}$ & NR & PE: $6(33 \%)$ & $\begin{array}{l}\text { Median GA: } 37.5 \text { week (36-39) } \\
\text { Median BW: } 2627 \mathrm{~g}(2136-3315) \\
\text { IUGR <10th: } 2 \text { (11\%) } \\
\text { IUGR<3rd: } 0 \\
\text { Apgar } 1 \text { min: } 8 \text { (7-9) } \\
\text { Apgar } 5 \text { min: } 9(9-9)\end{array}$ \\
\hline Su 2017 [29] & 116 & $\begin{array}{l}5(4 \%) \text { spont } \\
2(2 \%) \text { induc }\end{array}$ & $18(16 \%)$ & $90(78 \%)$ & $1(1 \%)$ & Preterm: $13(11 \%)$ & NR & $\begin{array}{l}\text { GHT: } 26 / 89(29 \%) \\
\text { Severe PE: } 12(10 \%) \\
\text { PtU >3.5 g/day: } \\
\text { 19/110 (17\%) }\end{array}$ & $\begin{array}{l}\text { CS: } 62(53 \%) \\
\text { Mean GA: } 37.8 \text { week } \pm 2.4 \\
\text { Mean BW: } 3035 \mathrm{~g} \pm 6688^{+} \\
\text {LBW <2500 g: } 16(17 \%)^{+} \\
\text {LBW <1500 g: } 3(3 \%)^{+}\end{array}$ \\
\hline
\end{tabular}


Table 3. Cont

\begin{tabular}{|c|c|c|c|c|c|c|c|c|c|}
\hline \multirow[b]{2}{*}{ Author Year [Ref] } & \multicolumn{5}{|c|}{ All Cases Considered } & \multicolumn{2}{|l|}{ Live Births Only } & \multirow{2}{*}{$\begin{array}{l}\text { All Cases } \\
\text { PE-HT }\end{array}$} & \multirow{2}{*}{$\begin{array}{l}\text { All Cases, or As Stated } \\
\text { Other }\end{array}$} \\
\hline & $\mathbf{P}$ & $\begin{array}{l}\text { Abort. } \\
\text { Spont. } \\
\text { Induced } \\
\end{array}$ & Still Birth & Live Birth & Neo. Death & $\begin{array}{l}\text { Preterm }<37 \text { weeks } \\
\text { Early }<34 \text { weeks } \\
\text { Extreme }<28 \text { weeks }\end{array}$ & NICU & & \\
\hline \multirow[b]{2}{*}{ Tocos 2017 [30] } & 33 cases & NA & $\mathrm{NA}$ & $33(100 \%)$ & NR & $\begin{array}{l}\text { Preterm: } 12(36 \%) \\
\text { Early: } 4(12 \%)^{+\dagger} \\
\text { Extreme: } 1(3 \%)^{+\dagger}\end{array}$ & $\mathrm{NR}$ & $\begin{array}{l}\text { GHT: } 7 / 24(29 \%)^{t++} \\
\text { PE: } 4 / 17(24 \%)^{++t}\end{array}$ & $\begin{array}{l}\text { CS: } 9(27 \%) \\
\text { LBW: } 10(30 \%) \\
\text { SGA <10th: } 4(12 \%)^{++} \\
\text {SGA <5th: } 1(3 \%)^{++}\end{array}$ \\
\hline & 1418 controls & NA & $\mathrm{NA}$ & $1418(100 \%)$ & $\mathrm{NR}$ & $\begin{array}{l}\text { Preterm: } 89(6 \%) \\
\text { Early: } 13(1 \%) \\
\text { Extreme: } 2(0.1 \%)\end{array}$ & NR & $\begin{array}{l}\text { HT: } 66(5 \%) \\
\text { PE: } 25(2 \%) \text { PtU: } 25(2 \%)\end{array}$ & $\begin{array}{l}\text { CS: } 379(27 \%) \\
\text { GA: } 39 \text { weeks }(25-42) \\
\text { BW: } 3232 \pm 476 \mathrm{~g} \\
\text { SGA <10th: } 120(8 \%) \\
\text { SGA <5th: } 45(3 \%)\end{array}$ \\
\hline Liu 2014 [31] & 69 & $8(12 \%)^{\S}$ & $2(3 \%)$ & $59(86 \%)$ & NR & Preterm: $7(10 \%)$ & NR & $\begin{array}{l}\text { Severe PE: } \\
6 \mathrm{~W}(10 \%)\end{array}$ & $\begin{array}{l}\text { CS: } 42(61 \%) \\
\text { LBW: } 8 / 59(14 \%) \\
\text { Mean BW: } 2972 \pm 654 \mathrm{~g}\end{array}$ \\
\hline Oh 2011 [32] & 52 & $\$ \S$ & NR & $\mathrm{NR}$ & NR & Preterm: $8(15.4 \%)$ & $4(7.7 \%)$ & $\begin{array}{l}\text { HT: }<8 \text { weeks } \\
31(60 \%)\end{array}$ & $\begin{array}{l}\text { CS: } 24(46.2 \%) \\
\text { LBW: } 13(25 \%)\end{array}$ \\
\hline Suetsugu $2011\left({ }^{* *}\right)[33]$ & 34 & NR & NR & NR & $1(3 \%)$ & NR & NR & Superimp. PE: 13 (38.2\%) & $\begin{array}{l}\text { BW negatively correlated with } \\
\text { glomerular sclerosis, sCr and BUN. }\end{array}$ \\
\hline Shimizu 2010 [34] & 29 & 0 & 0 & $29(100 \%)$ & 0 & $\begin{array}{l}0 \\
\text { Gestation } 38.0 \pm 2 \text { weeks }\end{array}$ & NR & No PE & $\begin{array}{l}\text { CS: } 5(17.2 \%) \\
\text { BW: } 2911.2 \pm 138.7 \mathrm{~g} \\
\text { LBW: } 0\end{array}$ \\
\hline Waness 2010 [35] & 12 & 0 & 0 & $12(100 \%)$ & NR & 0 & NR & $\begin{array}{l}\text { HT: } 12(100 \%) \\
\text { PE: } 3(25 \%) \\
\text { HELLP: } 1(8.3 \%)\end{array}$ & $\begin{array}{l}\text { CS: } 2 \text { (HELLP and PE) } \\
\text { BW: } 3.1 \mathrm{~kg} \\
\text { LBW: } 0 \\
\left.\text { Apgar: normal (1' } 1^{\prime} \text { and } 5^{\prime}\right)\end{array}$ \\
\hline Limardo 2010 [36] & 229 & $\begin{array}{l}15 \text { spon } \\
13 \text { indu }\end{array}$ & $5(2.2 \%)$ & $195(85 \%)$ & $1(0.4 \%)$ & Preterm: $20(10 \%)$ & NR & $\begin{array}{l}\text { HT: } 43 / 201(21 \%) \\
\text { PE: } 17 \mathrm{~W}(13 \%)\end{array}$ & $\begin{array}{l}\text { Mean BW: } 3039 \pm 610 \mathrm{~g} \\
\text { LBW: } 22 / 195(11 \%)\end{array}$ \\
\hline Donggyu $2010(*)[37]$ & 28 & NR & NR & $\mathrm{NR}$ & NR & NR & NR & $\begin{array}{l}\text { PE: } 4 \mathrm{~W} \text { (of } 5 \text { with } \mathrm{sCr} \\
>2.0 \mathrm{mg} / \mathrm{dL} \text { ) }\end{array}$ & NR \\
\hline Ronkainen 2006 [38] & 22 & $\begin{array}{l}\text { At least } 2 \\
\text { spont }\end{array}$ & NR & 20 & NR & $\begin{array}{l}\text { Preterm: at least } 6(30 \%) \\
\text { Extreme: at least } 2(10 \%)\end{array}$ & NR & $\begin{array}{l}\text { HT: } 10(46 \%) \\
\text { Severe PE: } 1 \mathrm{~W}(10 \%) \\
\text { PtU: } 12(55 \%)\end{array}$ & $\begin{array}{l}6(30 \%) \text { of } 20 \text { live born infants from } \\
\text { mothers with HT and/or } \\
\text { proteinuria premature }\end{array}$ \\
\hline Ronkainen 2002 [39] & 23 & NR & NR & NR & NR & NR & NR & HT and/or PtU: 16 (70\%) & $\mathrm{NR}$ \\
\hline Summary data & 729 & 45/485 (9.3\%) & $25 / 473(5.3 \%)$ & $\begin{array}{l}456 / 528 \\
(86.3 \%)\end{array}$ & $5 / 426(1.2 \%)$ & $\begin{array}{l}\text { Preterm } 95 / 608(15.6 \%) \\
\text { Early: } 18 / 135(13.3 \%) \\
\text { Extreme: } 3 / 53(5.6 \%)\end{array}$ & $4 / 52(7.7 \%)$ & $\begin{array}{l}\text { PIH: } 98 / 348(28.2 \%) \\
\text { PE: } 79 / 523(15.1 \%)\end{array}$ & $\begin{array}{l}\text { CS: 144/311 (46.3\%) } \\
\text { LBW: } 85 / 530(16.0 \%) \\
\text { IUGR/SGA <10th: } 12 / 115(10.4 \%)\end{array}$ \\
\hline
\end{tabular}

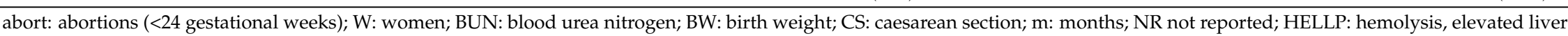
enzymes, low platelets syndrome; HT: hypertension; LBW: low birth weight ( $<2500 \mathrm{~g})$; NA: not applicable, only included births $>20$ weeks or only live births; NICU: neonatal intensive care unit; NR: not reported; PE: preeclampsia; P: pregnancies; PtU: proteinuria; sCr: serum creatinine; ${ }^{*}$ Abstract only $\left({ }^{* *}\right)$ paper in Japanese, abstract used, ${ }^{\dagger}$ data on $86 / 116$ pregnancies, ${ }^{\dagger+}$ not mentioned in article, additional information available in original database, ${ }^{+++}$calculated in $\mathrm{W}$ without baseline hypertension and/or proteinuria, ${ }^{\S}$ spontaneous abortion: 1 ( $2 \%$ ); induced abortion: 1 (2\%); embryo damage $3(4 \%)$; fetal malformation $3(4 \%)$., $\$ \$ 14$ abortions in 80 women, not included in final analyses of the study. Note: if not otherwise clarified, numbers of cases with hypertension, $\mathrm{PE}$ and/or PtU were counted separately. 
Table 4. IgA Nephropathy. Kidney function and other maternal outcomes in the case series reporting on them.

\begin{tabular}{|c|c|c|c|}
\hline Author Year [Ref] & Age (years) & Kidney Function at Baseline & Other Maternal Outcomes and Main Results \\
\hline Park 2017 [27] & 28 (24-31) (cases) & $\begin{array}{l}\text { eGFR: } 80.0 \text { (61.0-105.6) } \\
\text { sCr: } 0.90 \mathrm{mg} / \mathrm{dL}(0.70-1.00) \\
\text { PtU: } 1.09 \mathrm{~g} / \text { day }(0.46-2.02) \\
\text { HT: } 36(61 \%)\end{array}$ & $\begin{array}{l}\text { Renal survival rate with gestational complications: } 55.3 \% \text { at } 10 \mathrm{y} ; 46.1 \% \text { at } 20 \text { years } \\
\text { Renal survival rate without gestational complications: } 97.3 \% \text { at } 10 \text {; } 97.3 \% \text { at } 20 \text { years } \\
\text { Obstetric complications (PE, LBW and/or preterm birth), not pregnancy itself, associated with CKD } \\
\text { progression, especially if eGFR }<60 \text {, preexisting HT and PtU }>1 \text { g/day (all significant) }\end{array}$ \\
\hline O'Shaughnessy 2017 [28] & $31.3(23.0-33.8)$ & $\begin{array}{l}\text { eGFR: } 72(61-90)(9 / 18 \mathrm{P}) \\
\text { sCr: } 1.0 \mathrm{mg} / \mathrm{dL}(0.8-1.2)(9 / 18 \mathrm{P}) \\
\text { PtU spot: } 1.3 \mathrm{~g}(0.9-4.1)(8 / 18 \mathrm{P})\end{array}$ & $\begin{array}{l}\geq 200 \% \text { increase } \mathrm{PtU}(2-12 \mathrm{~m} \text { postpartum): } 2 / 6(33.3 \%) \\
\geq 150 \% \text { increase } \mathrm{sCr}(2-12 \mathrm{~m} \text { postpartum): } 1 / 8(12.5 \%) \\
\text { ESRD } 1 \text { year postpartum: } 2(11.1 \%) \S \\
\text { Active IgAN during pregnancy: } 12(66.7 \%) \text {. Dialysis during pregnancy: } 0\end{array}$ \\
\hline \multirow{2}{*}{ Su 2017 [29] } & $27.2 \pm 3.5$ (cases) & $\begin{array}{l}\text { eGFR: } 102.6 \pm 23.9 \\
\text { PtU: } 1.04 \text { g/day }(0.03-7.25) \\
\text { HT: } 15(14 \%) \\
\text { Follow up: } 67 \pm 34 \text { months }\end{array}$ & $\begin{array}{l}\text { Persistent HT postpartum: } 12 / 89(13 \%) \text {. Irreversible PtU worsening: } 7(6 \%) \\
\text { PtU at pregnancy start or first trimester: risk factor for severe PE and infant loss } \\
\text { ESRD: } 4(4 \%))^{\S} \text { ESRD/ }>50 \% \text { decrease eGFR: } 7(7 \%) \\
\text { Significant decrease kidney function after pregnancy in CKD stage 3-4 only }\end{array}$ \\
\hline & $28.7 \pm 6.3$ (controls) & $\begin{array}{l}\text { eGFR: } 94.5 \pm 26.7 \\
\text { PtU: } 1.29 \mathrm{~g} / \text { day }(0.02-11.78) \\
\text { HT: } 52(17 \%) \\
\text { Follow up: } 65 \pm 34 \text { months }\end{array}$ & $\begin{array}{l}\text { ESRD: } 18(6 \%)^{\S} \\
\text { ESRD/>50\% decrease eGFR: } 31(10 \%)\end{array}$ \\
\hline \multirow[t]{2}{*}{ Tocos 2017 [30] } & $31.9 \pm 5.2$ (cases) & $\begin{array}{l}\text { eGFR: } 89.9 \pm 32.7 \\
\text { sCr: } 0.87 \mathrm{mg} / \mathrm{dL}(0.50-2.88) \\
\text { PtU } \geq 0.5 \mathrm{~g} / \text { day: } 13(41 \%) \\
\text { HT: } 9(27 \%)\end{array}$ & $\begin{array}{l}\text { Worsening CKD stage during pregnancy: } 1 / 33(3 \%) \$ \S \\
\text { Increased risk of PE but not of preterm delivery suggests late maternal PE }\end{array}$ \\
\hline & $31.2 \pm 5.5$ (controls) & HT: none & \\
\hline \multirow[t]{2}{*}{ Liu 2014 [31] } & $27.3 \pm 3.6$ (cases) & $\begin{array}{l}\text { eGFR: } 102.3 \pm 21.9 \\
\text { PtU: } 1.27(0.06-7.25) \text { g/day } \\
\text { HT: } 7(11 \%)\end{array}$ & $\begin{array}{l}\text { HT after pregnancy } 8(13 \%) \text {; MAP during follow up } 86.4 \pm 8.6 \\
\text { Kidney disease progression: } 4(6 \%) \text {; decrease eGFR }>50 \%: 3(5 \%) \text {; ESRD: } 1(2 \%) \S \\
\text { Mean change eGFR: }-2.5 \mathrm{~mL} / \mathrm{min}(-6.7 \text { to } 0.06) \\
\text { PtU during follow up: } 0.67 \mathrm{~g} / \text { day }(0.10-6.72) \\
\text { Proteinuria in pregnancy borderline significant for adverse pregnancy outcomes }\end{array}$ \\
\hline & $27.8 \pm 4.4$ (controls) & $\begin{array}{l}\text { eGFR: } 103.4 \pm 20.8 \\
\text { PtU: } 1.09(0.06-8.37) \text { g/day } \\
\text { HT: } 4(6 \%)\end{array}$ & $\begin{array}{l}\text { MAP during follow up } 85.4 \pm 7.3 ; \text { Kidney disease progression: } 6(10 \%) \\
\left.\text { decrease eGFR }>50 \%: 4(7 \%) \text {; ESRD: } 2(3 \%){ }^{\S} \text { Mean change eGFR }-2.4-7.1 \text { to } 2.4\right) \mathrm{mL} / \mathrm{min} \\
\text { PtU during follow up: } 0.68 \text { (0.07-4.30) g/day }\end{array}$ \\
\hline Oh 2011 [32] & $30.5(25.0-39.0)$ & $\begin{array}{l}\text { eGFR: } 91.2(24.1-157.0) \mathrm{mL} / \mathrm{min} \\
\text { MAP: } 89.6-99.3 \mathrm{mmHg}\end{array}$ & $\begin{array}{l}\text { eGFR after delivery } 77.8(19.8-150.0) \\
\text { Median } \triangle \text { GFR with } \leq 30 \% \text { reduction proteinuria prior to conception: } 13 \% \\
\text { Median } \triangle \text { GFR with }>30 \% \text { reduction proteinuria prior to conception: } 8.7 \% \\
\text { MAP during pregnancy } 96.7-102 \\
\text { Significant increase } \mathrm{s} C \mathrm{Cr}(0.8-1.0 \mathrm{mg} / \mathrm{dL}) \text { and } \mathrm{PtU}(0.7-1.5 \mathrm{~g} / \mathrm{g}) \text { after delivery }\end{array}$ \\
\hline Suetsugu $2011\left(^{*}\right)[33]$ & NR & NR & $\begin{array}{l}\text { Superimposed PE: preconception SBP, sCr, BUN higher; } \mathrm{CCr} \text { and eGFR lower } \\
\text { Delivery: sCr, BUN, uric acid higher; } \mathrm{CC} \text { and eGFR lower (significant) } \\
\text { At delivery correlation between BP and histological severity, proteinuria and sCr }\end{array}$ \\
\hline
\end{tabular}


Table 4. Cont.

\begin{tabular}{|c|c|c|c|}
\hline Author Year [Ref] & Age (years) & Kidney Function at Baseline & Other Maternal Outcomes and Main Results \\
\hline \multirow[t]{2}{*}{ Shimizu 2010 [34] } & $27.3 \pm 4.0$ (cases) & $\begin{array}{l}\text { eGFR mL/min } \\
\text { CKD1: } 97.3 \pm 9.4 \\
\text { CKD2: } 74.1 \pm 4.5 \\
\text { CKD3: } 54.4 \pm 11.6\end{array}$ & 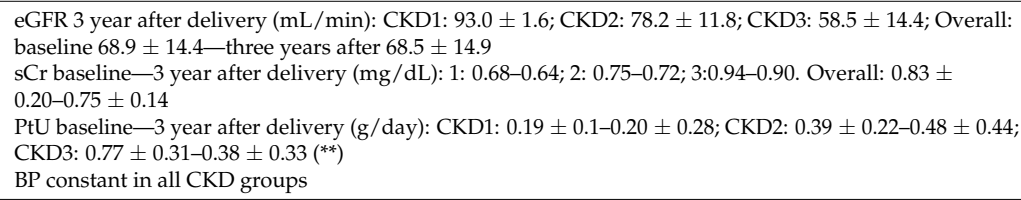 \\
\hline & $28.1 \pm 5.1$ (controls) & eGFR: $70.9 \pm 20.7$ & $\begin{array}{l}\text { eGFR after } 3 \text { years }(\mathrm{mL} / \mathrm{min}): 68.6 \pm 14.4 \\
\text { sCr baseline-after } 3 \text { years }(\mathrm{mg} / \mathrm{dL}): 0.8 \pm 0.15-0.88 \pm 0.16 \\
\text { PtU baseline-after } 3 \text { years }(\mathrm{g} / \mathrm{day}): 0.85 \pm 0.65-0.40 \pm 0.26 \\
\text { No new onset hypertension }\end{array}$ \\
\hline Waness 2010 [35] & 28.6 & $\begin{array}{l}\text { CCr } 88.6 \mathrm{~mL} / \mathrm{min} \\
\text { SCr: } 0.99 \mathrm{mg} / \mathrm{dL} \\
\text { BP: } 128.2 / 82.1 \mathrm{mmHg} \\
\text { PtU } 535.2 \mathrm{mg} / \mathrm{day}\end{array}$ & $\begin{array}{l}\text { In } 3 \text { rd trimester: BP } 163.7 / 90.3 \mathrm{mmHg} \\
\mathrm{PtU} 2179.2 \mathrm{mg} / \text { day } \\
\mathrm{CCr} 77.4 \mathrm{~mL} / \mathrm{mins} \\
\mathrm{Cr} 84.3 \mathrm{mmol} / \mathrm{L}\end{array}$ \\
\hline \multirow[t]{2}{*}{ Limardo 2010 [36] } & $26.72 \pm 4.27$ (cases) & $\begin{array}{l}\text { sCr } 0.87 \pm 0.15 \\
\text { CCr } 92 \pm 17 \\
\text { PtU } 1.0(0-6) \text { g/day } \\
\text { HT: } 27(20 \%)\end{array}$ & $\begin{array}{l}\text { After } 10 \text { years: } 36 \% \text { on steroids and/or immuno-depressors; } 61 \% \text { on ACEI or ARBs } \\
\text { Significant CCr decrease }(-1.2 \mathrm{~mL} / \mathrm{min} / \text { year) in women with PtU }>1 \mathrm{~g} / \text { day at diagnosis, not modified by } \\
\text { number of pregnancies, hypertension, PE } \\
\text { Doubling of sCr in } 13(9.6 \%) \text {; start of dialysis in } 3(3.4 \%){ }^{\S} \text {; new-onset HT in } 34(31 \%) \text { of } 109 \text { previously } \\
\text { normotensive women }\end{array}$ \\
\hline & $26.19 \pm 5.15$ (controls) & $\begin{array}{l}\mathrm{sCr} 0.86 \pm 0.16 \\
\mathrm{CCr} 89 \pm 18 \\
\text { PtU } 0.5(0-7.6) \mathrm{g} / \text { day } \\
\text { HT: } 10(11 \%)\end{array}$ & $\begin{array}{l}\text { After } 10 \text { years: } 29 \% \text { on steroids and /or immune-depressors; } 47 \% \text { on ACEI or ARBs } \\
\text { Doubling of Cr in } 7(8 \%) \text {; start of dialysis in } 2(1.5 \%) \text { \$; new-onset HT in } 16 \text { of } 77 \text { (21\%) previously } \\
\text { normotensive women }\end{array}$ \\
\hline Donggyu $2010 *[37]$ & NR & NR & $\begin{array}{l}\text { PE in } 4 \text { of } 5 \text { women with } \mathrm{sCr}>2.0 \mathrm{mg} / \mathrm{dL} \text { at delivery } \\
\text { ESRD within } 2 \text { years in } 2 / 2 \mathrm{~W} \text { with } \mathrm{sCr}>2.5 \mathrm{mg} / \mathrm{dL} \text { in postpartum } \\
\text { All women with } \mathrm{sCr}<2.5 \mathrm{mg} / \mathrm{dL} \text { in postpartum had stable sCr } 3 \text { year after delivery }\end{array}$ \\
\hline Ronkainen 2006 [38] & NR & NR & $\begin{array}{l}\text { ESRD } 2.6 \text { year after delivery in } 1 \text { hypertensive woman with slightly impaired renal function before } \\
\text { pregnancy } \$\end{array}$ \\
\hline Ronkainen 2002 [39] & NR & NR & $\begin{array}{l}\mathrm{HT} \text { or PtU in pregnancy: } 9(64.3 \%) \text {, of whom } 5 \text { reported poor outcome (not specified); no poor outcome } \\
\text { reported in women without } \mathrm{HT} \text { or PtU in pregnancy }\end{array}$ \\
\hline Summary data baseline & \multicolumn{3}{|c|}{$\begin{array}{l}\text { GFR or } \mathrm{CCr}>100 \mathrm{~mL} / \mathrm{min} \text { in } 2 / 9 \text { study reporting on this item } \\
\text { GFR }<100 \mathrm{~mL} / \mathrm{min} \text { in } 7 / 9 \text { studies } \\
\text { PtU } \geq 0.5 \mathrm{~g} / \text { day in } 6 / 7 ;<0.5 \mathrm{~g} / \text { day in } 1 / 7 \text { studies } * * *) \\
\text { Hypertension in } 11-61 \% \text { in } 4 \text { studies, in other not clearly defined at baseline }\end{array}$} \\
\hline Summary data progression & \multicolumn{3}{|c|}{$\begin{array}{l}\text { ESRD: } 11 / 330(3.3 \%) \text { cases vs } 22 / 458(4.8 \%) \text { controls, reported on by } 5 \text { studies of whom } 3 \text { provided a control group } \$ \\
\text { Park: no significant difference between cases and non-pregnant controls. Significant better renal survival in cases without vs } \\
\text { complications vs non pregnant controls } \\
\text { Su: no significant difference in incidence ESRD or eGFR decrease between cases and non-pregnant controls } \\
\text { Liu: no difference between cases and non-pregnant controls over follow-up } \\
\text { Shimizu: no difference in eGFR decrease between pregnancy and non-pregnancy } \\
\text { Limardo: no significant difference in all outcomes (start of dialysis, doubling of serum creatinine, new onset hypertension) }\end{array}$} \\
\hline
\end{tabular}

BP: blood pressure; CCr: creatinine clearance; (e)GFR: (estimated) glomerular filtration rate; ESRD: end-stage renal disease; HT: hypertension; MAP: mean arterial pressure; NR: not reported; P: pregnancies; PE: preeclampsia; PtU: proteinuria; sCr: serum creatinine. * Abstract only $\left({ }^{*}\right)$ article in Japanese, abstract used. ${ }^{* *}$ discrepant data between the various CKD stages and overall, as for proteinuria: Overall: $0.86 \pm 0.80-0.56 \pm 0.48 . \$$ used in meta-analysis despite of difference in follow-up (1 to 10 years); $\$ \$$ not mentioned in article, additional information available in original database. 
Table 5. IgA Nephropathy. Case reports: baseline data.

\begin{tabular}{|c|c|c|c|c|c|}
\hline Author Year [Ref] & Country & Age (years) & sCr-GFR-PtU & Other Data at Referral & Main Drugs in Pregnancy \\
\hline \multirow{3}{*}{ Kaul 2016 * [40] } & \multirow{3}{*}{ India } & NR & NR & IgAN new-onset & Steroids, fish oil \\
\hline & & NR & NR & IgAN new-onset & Steroids, fish oil \\
\hline & & NR & NR & IgAN new-onset & Steroids, fish oil \\
\hline $\operatorname{Lim} 2016 *$ [41] & USA & NR & NR & $\begin{array}{l}\text { IgAN (diagnosed several } \\
\text { years postpartum) }\end{array}$ & NR \\
\hline Sun 2015 [42] & China & 26 & PtU 1-2+ & IgAN new-onset & NR \\
\hline Nagai 2015 [43] & Japan & 37 & PtU postpartum & HSPN postpartum & NR \\
\hline Liang $2015 *$ [44] & USA & 32 & PtU 2 g/day & IgAN new-onset & NR \\
\hline Zand 2014 [45] & USA & 18 & $\mathrm{sCr} 1.8 \mathrm{mg} / \mathrm{dL}$ & IgAN & NR \\
\hline Cornelis 2013 [46] & The Netherlands & 21 & $\mathrm{CCr} 20-25 \mathrm{~mL} / \mathrm{min}$ & IgAN & Methyldopa, labetalol, EPO, thyroid hormones, oral iron \\
\hline Hou 2013 [47] & USA & 28 & $\begin{array}{l}\mathrm{CCr} 79 \mathrm{~mL} / \mathrm{min} \\
\mathrm{PtU} 1.13 \mathrm{~g} / \text { day }\end{array}$ & IgAN new-onset & $\begin{array}{l}\text { Methyldopa, labetalol, hydralazine, magnesium (31 week), steroid } \\
\text { prophylaxis ( } 31 \text { week) }\end{array}$ \\
\hline \multirow{3}{*}{ Goifrè 2007 [48] } & \multirow{3}{*}{ Italy } & 25 & $\begin{array}{l}\mathrm{sCr} 2.2 \mathrm{mg} / \mathrm{dL} \\
\mathrm{PtU} 1 \text { g/day }\end{array}$ & \multirow{3}{*}{ IgAN } & ASA, oral iron, vitamins, vaginal dinoprostone gel (36 week) \\
\hline & & 30 & $\mathrm{sCr} 8 \mathrm{mg} / \mathrm{dL}$ & & EPO, vit D, calcium carbonate, ritodrin (29 week) \\
\hline & & 32 & HD & & $E P O$, vit $D$, calcium carbonate, ritodrin (30 week) \\
\hline Tanno 2007 [49] & Japan & 31 & $\mathrm{~s} C \mathrm{Cr} 0.8 \mathrm{mg} / \mathrm{dL}$ & $\begin{array}{l}\text { HSPN recurrence in renal } \\
\text { allograft }\end{array}$ & $\begin{array}{l}\text { Methyldopa, amlodipine } \\
\text { Immunosuppressors (not clear) }\end{array}$ \\
\hline Barquero-Romero 2006 [50] & Spain & 36 & $\begin{array}{l}\mathrm{sCr} 0.50 \mathrm{mg} / \mathrm{dL} \\
\mathrm{PtU} 1+\end{array}$ & HSPN new-onset & Methylprednisolone \\
\hline Koizumi 2004 [51] & Japan & 30 & $\mathrm{PtU}+$ & HSPN new-onset & Low dose oral steroids for 3 week \\
\hline Cusi 2003 [52] & Italy & 29 & $\begin{array}{l}\mathrm{sCr} 1.5 \mathrm{mg} / \mathrm{dL} \\
\mathrm{PtU} 1.2 \mathrm{~g} / \text { day }\end{array}$ & IgAN & Methyldopa, nifedipine, clonidine, EPO, steroid prophylaxis (26 week) \\
\hline Amir $2002\left(^{*}\right)[53]$ & Saudi Arabia & NR & $\begin{array}{l}\mathrm{sCr} 2.7 \mathrm{mg} / \mathrm{dL} \\
\mathrm{PtU} 5.4 \mathrm{~g} / \mathrm{day}\end{array}$ & IgAN with P-ANCA & Cyclophosphamide, prednisone \\
\hline Summary data & & $28.8(18-37)$ & $\begin{array}{l}\mathrm{sCr}<1.0 \mathrm{mg} / \mathrm{dL}: 2 / 9(22.2 \%) \\
\mathrm{SCr} \geq 1.0 \mathrm{mg} / \mathrm{dL}: 5 / 9(55.6 \%) \\
\mathrm{CCr}<90 \mathrm{~mL} / \mathrm{min}: 2 / 9(22.2 \%) \\
\mathrm{CCr} \geq 90 \mathrm{~mL} / \mathrm{min}: 0 \\
\mathrm{PtU}>=0.5 \mathrm{~g} / \text { day: } 5 / 5(100 \%) \\
\text { reporting quantitatively }\end{array}$ & $\begin{array}{l}\text { IgAN: } 12 \text { (new-onset: 6) } \\
\text { HSPN: } 4 \text { (new-onset: 3) }\end{array}$ & $\begin{array}{l}\text { Antihypertensive agent: } 4 / 10 \\
\text { Immunosuppressors: } 4 / 10\end{array}$ \\
\hline
\end{tabular}

ASA: acetyl salicylic acid; CCr: creatinine clearance; EPO: Erythropoietin; GFR: Glomerular filtration rate; h: hours; HSPN: Henoch-Schönlein purpura nephropathy; IgAN: IgA nephropathy; i.v.: intravenous; methyldopa: alpha-methyldopa; NR: Not reported; PtU: proteinuria; sCr: serum creatinine; vit: vitamin; w: week. ${ }^{*}$ Abstract only $\left({ }^{*}\right)$ abstract used. 
Table 6. IgA Nephropathy. Case reports: fetal outcomes.

\begin{tabular}{|c|c|c|c|c|c|c|c|c|c|c|}
\hline Author Year [Ref] & Pts & GW & Parity & Delivery & Indication for Delivery & NICU & $\begin{array}{l}\text { APGAR 1-5 min } \\
\text { Infant Outcomes }\end{array}$ & Sex & Weight (g) & Centile * \\
\hline Kaul 2016* [40] & 3 & NR & NR & NR & NR & NR & All live births & NR & NR & NR \\
\hline $\operatorname{Lim} 2016$ * [41] & 1 & NR & NR & NR & NR & NR & NR & NR & NR & NR \\
\hline Sun 2015 [42] & 1 & 40 & Primi & CS & NR & NR & NR & NR & NR & NR \\
\hline Nagai 2015 [43] & 1 & At term & NR & Vaginal & None & NR & NR & NR & NR & NR \\
\hline Liang $2015 *$ [44] & 1 & $36+5$ & $\begin{array}{l}\text { Gravi } 7 \\
\text { Para } 1 \\
\text { P1051 }\end{array}$ & $\begin{array}{l}\text { Vaginal } \\
\text { induced }\end{array}$ & $\begin{array}{l}\text { Presumed superimposed } \\
\text { PE }\end{array}$ & NR & NR & $\mathrm{F}$ & NR & NR \\
\hline Zand 2014 [45] & 1 & 32 & NR & Vaginal & None & NR & Healthy & $\mathrm{M}$ & NR & NR \\
\hline Cornelis 2013 [46] & 1 & 36 & Primi & $\begin{array}{l}\text { Vaginal } \\
\text { assisted }\end{array}$ & Sudden HT & YES & $\begin{array}{l}9 \text { and } 10 \\
\text { Wet lung syndrome, NICU non-invasive } \\
\text { ventilatory support } 1 \text { day. Discharged } 8 \\
\text { days later. }\end{array}$ & $\mathrm{M}$ & 2480 & 25 \\
\hline Hou 2013 [47] & 1 & 31 & $\begin{array}{l}\text { Gravi } 2 \\
\text { Para } 0\end{array}$ & CS & $\mathrm{PE}$, failed induction & NR & $\begin{array}{l}3 \text { and } 8 \\
\text { Normal development } 11 \text { years later. }\end{array}$ & $\mathrm{F}$ & 1596 & 64 \\
\hline \multirow{3}{*}{ Goifrè 2007 [48] } & \multirow{3}{*}{1} & 38 & $\begin{array}{l}\text { Gravi } 1 \\
\text { Para } 0 \\
\end{array}$ & Vaginal & None & YES & $\begin{array}{l}8 \text { and } 9 \\
\text { NICU, discharged } 20 \text { days later. }\end{array}$ & $\mathrm{M}$ & 3150 & 45 \\
\hline & & 33 & $\begin{array}{l}\text { Gravi } 2 \\
\text { Para } 1\end{array}$ & CS & NR & YES & $\begin{array}{l}7 \text { and } 8 \\
\text { NICU for RD (ventilatory support for } 6 \\
\text { h); discharged } 20 \text { days later. }\end{array}$ & $\mathrm{M}$ & 2190 & 65 \\
\hline & & 33 & $\begin{array}{l}\text { Gravi } 3 \\
\text { Para } 2\end{array}$ & CS & NR & YES & $\begin{array}{l}5 \text { and } 8 \\
\text { NICU for RD (ventilatory support for } 2 \\
\text { days); discharged } 30 \text { days later. }\end{array}$ & $\mathrm{M}$ & 2500 & 90 \\
\hline Tanno 2007 [49] & 1 & 28 & NR & CS & $\begin{array}{l}\text { Impaired umbilical flow } \\
\text { and fetal growth }\end{array}$ & NR & No obvious anomalies & NR & 999 & $39 * *$ \\
\hline $\begin{array}{l}\text { Barquero-Romero } \\
2006[50]\end{array}$ & 1 & 39 & Multi & Vaginal & None & NR & Healthy at $3 \mathrm{~m}$ follow up & $\mathrm{M}$ & 3380 & 41 \\
\hline Koizumi 2004 [51] & 1 & 40 & Primi & Vaginal & None & NR & Healthy & $\mathrm{M}$ & 2986 & 11 \\
\hline Cusi 2003 [52] & 1 & 31 & $\mathrm{NR}$ & Vaginal & None & NR & $\begin{array}{l}8 \text { and } 9 \\
\text { Healthy }\end{array}$ & $\mathrm{F}$ & 1626 & 68 \\
\hline Amir $2002\left(^{*}\right)[53]$ & 1 & NR & NR & NR & NR & NR & NR & NR & NR & NR \\
\hline Summary data & $\begin{array}{l}16(18 \\
\text { cases })\end{array}$ & $\begin{array}{l}<37 \mathrm{w}: 8 / 13 \\
(61.5 \%) \\
\geq 37 \mathrm{w}: 5 / 13 \\
(38.5 \%)\end{array}$ & $\begin{array}{l}\text { Primi: } 4 / 9 \\
(44.4 \%) \\
\text { Multi: } 5 / 9 \\
(55.6 \%)\end{array}$ & $\begin{array}{l}\text { Vaginal: } \\
8 / 13(61.5 \%) \\
\text { CS: } 5 / 13 \\
(38.5 \%)\end{array}$ & $\begin{array}{l}\text { Maternal complications: } \\
3 / 10(30 \%) \\
\text { Fetal complications: } 1 / 10 \\
(10 \%) \\
\text { None: } 6 / 10(60 \%)\end{array}$ & $\begin{array}{l}\text { All rep } \\
\text { reporte }\end{array}$ & $\begin{array}{l}\text { ases had favorable outcomes; NICU } \\
\text { ases }\end{array}$ & $\begin{array}{l}\text { F: } 3 / 10 \\
(30 \%) \\
\text { M: } \\
7 / 10 \\
(70 \%)\end{array}$ & $\begin{array}{l}<2500 \mathrm{~g}: 5 / 9 \\
(55.6 \%) ; \\
<1500 \mathrm{~g}: 1 / 9 \\
(11.1 \%)\end{array}$ & $\begin{array}{l}\text { AGA: } 9 / 9 \\
(100 \%) \\
\text { (calculated } \\
\text { upon INeS } \\
\text { charts) }\end{array}$ \\
\hline
\end{tabular}

AGA: appropriate for gestational age; CS: cesarean; d: days; F: female; Gravi: gravidity; GW: Week of gestation; h: hours; HT: hypertension; LGA: large for gestational age; M: male; m: months; Multi: multipara; NICU: neonatal intensive care unit; NR: not reported; Para/P: parity; PE: preeclampsia; Primi: primipara; Pts: Patients; RD: respiratory distress; w: weeks. * Abstract only $\left(^{*}\right)$ abstract used ${ }^{*}$ Centiles calculated according to the INeS charts (reference). If parity or sex are not specified: male sex and primipara considered. 
Table 7. IgA Nephropathy. Case reports: maternal outcomes.

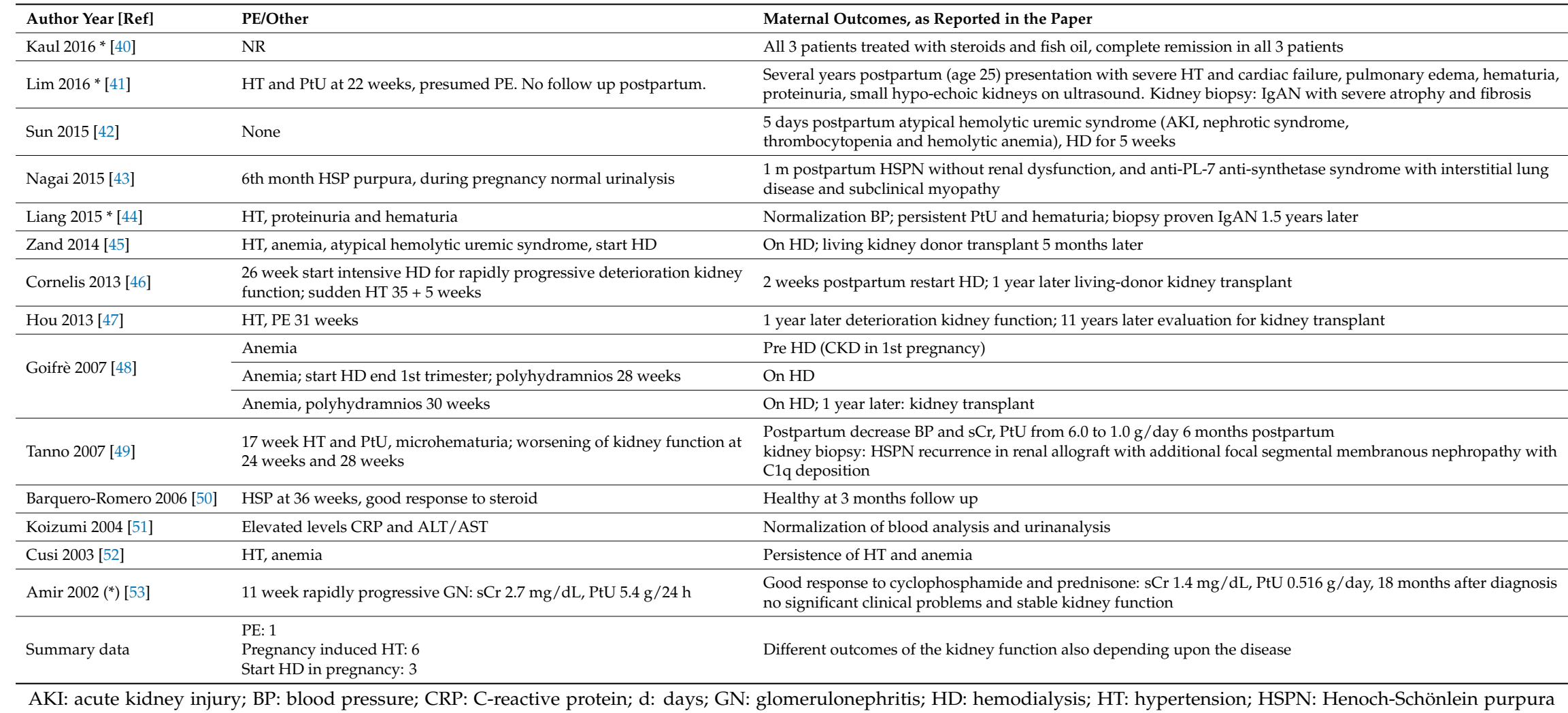

nephropathy; IgAN: IgA nephropathy; NR: Not reported; PE: preeclampsia; PtU: proteinuria; * Abstract only $\left({ }^{*}\right)$ abstract used. 


\subsection{Meta-Analysis}

Kidney Function

Figure 2 reports on ESRD and the start of renal replacement therapy in patients with and without pregnancy. The incidence was low in the five studies reporting on this outcome: 11/330 patients versus 22/458 controls, and there was no significant difference found between the two groups (OR 0.68 ; CI $0.28-1.65)$.

\section{Odds ratio for ESRD or start of renal replacement therapy in pregnant women with IgA nephropathy versus non-pregnant controls with IgA nephropathy.}

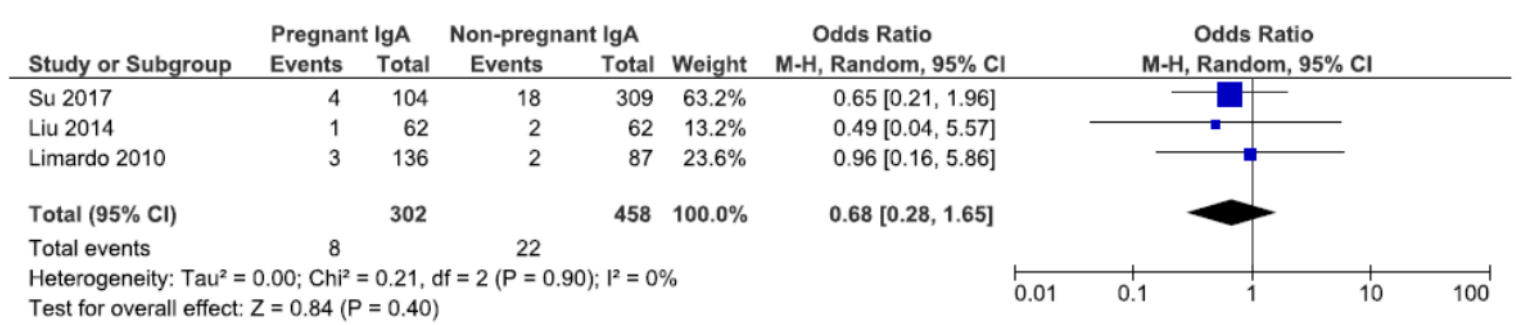

Figure 2. Odds ratio for end-stage renal disease in patients with IgA nephropathy, with or without pregnancy. Legend: ESRD: end stage renal disease. CI: confidence intervals.

These reassuring data have to be contextualized to patients with good renal function, and it has to be taken into account that time of follow up differed notably between the studies (ranging from 1 to 10 years postpartum), which reduces the clinical value of this comparison (Figure 2).

\subsection{Pregnancy Related Outcomes}

The risk for adverse pregnancy-related outcomes was higher in IgA patients than in the low-risk cohort (Figure 3).

While the incidence of caesarean sections and the incidence of small for gestational age babies were not significantly different in IgA nephropathy and in the low-risk control group, the odds ratio for preterm delivery, PE and PIH were significantly higher for women with IgA nephropathy (Figure 3).

Odds ratio (OR) for PE and PIH were particularly high: PE: OR 11.80; CI 7.53-18.48, PIH OR 10.39; CI 5.45-19.80. Conversely, the risk of preterm birth was about threefold that of the low-risk population (OR 3.37; CI 1.91-5.95), while the risk of neonates with low birth weight is about twice as high (OR 2.36; CI 1.52-3.66). 
Odds ratio of pregnancy related outcomes in IgA nephropathy versus low-risk controls: PE (a), hypertension* (b), preterm delivery (c), early preterm delivery (d), low birth weight (e), SGA - IUGR (f), caesarean section (g), stillbirth (h).

a

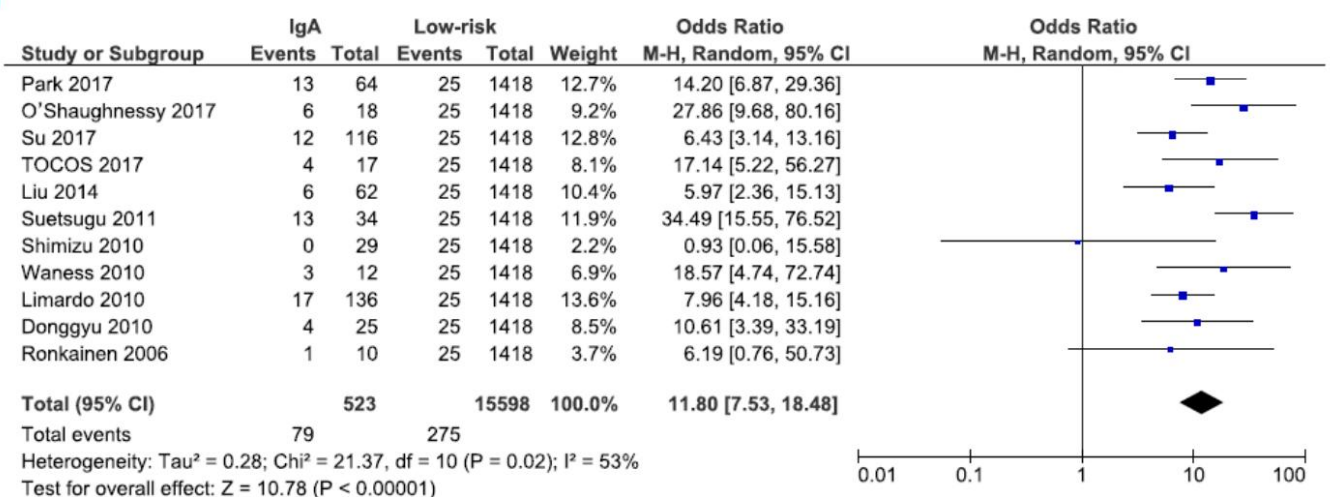

b

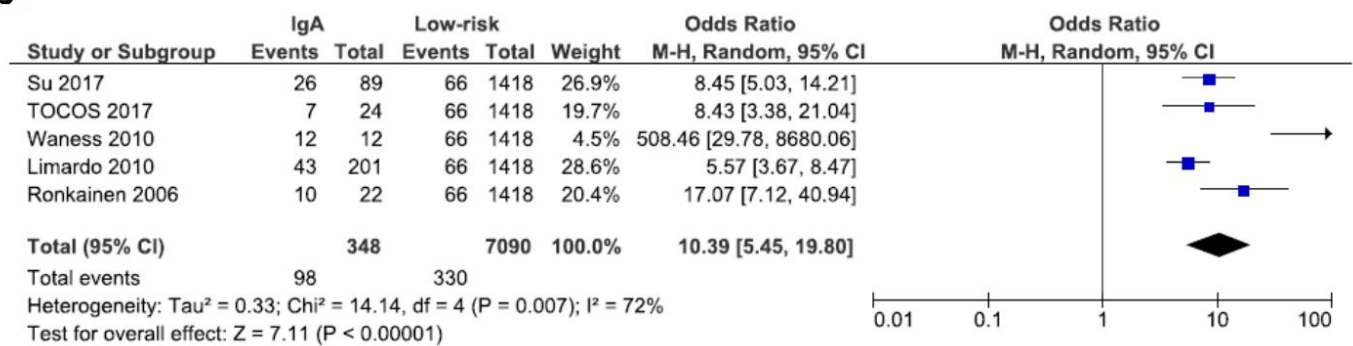

C

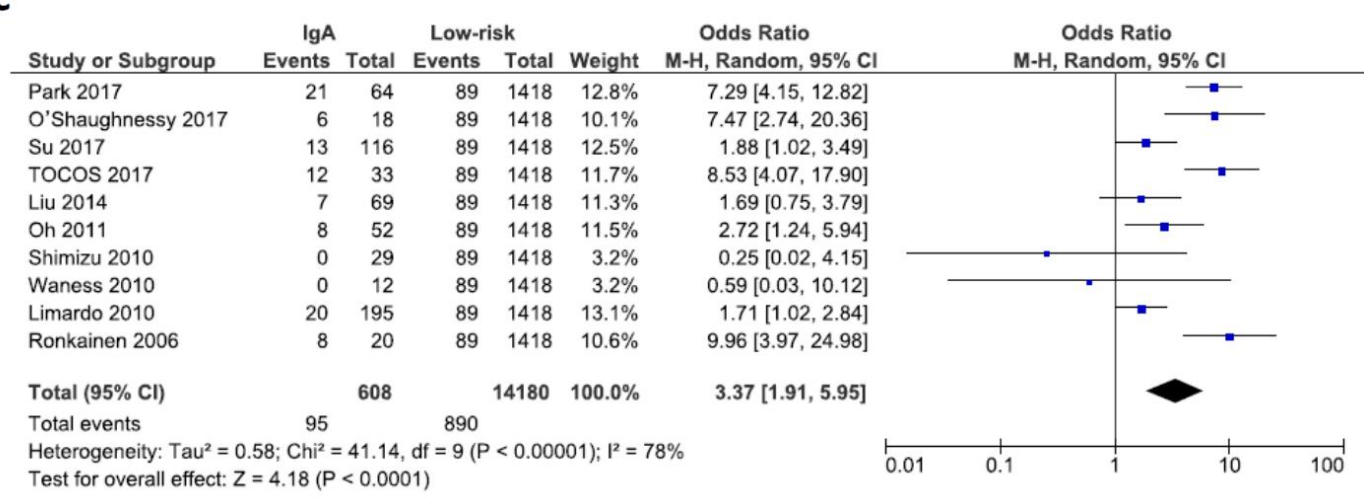

d

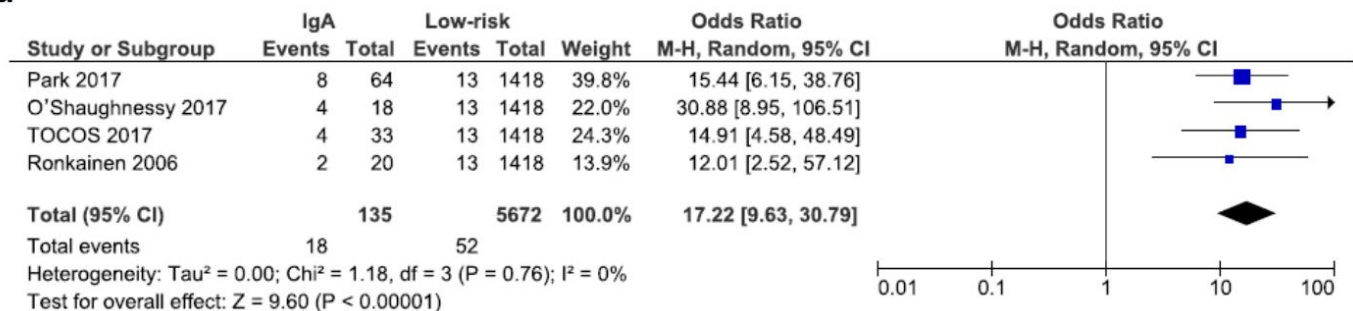

Figure 3. Cont. 


\section{e}

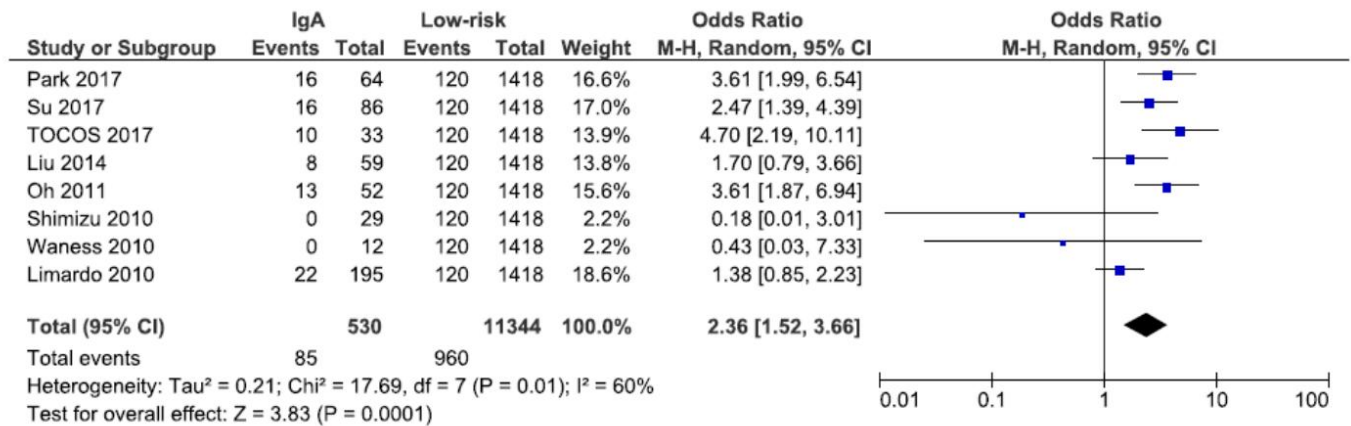

f

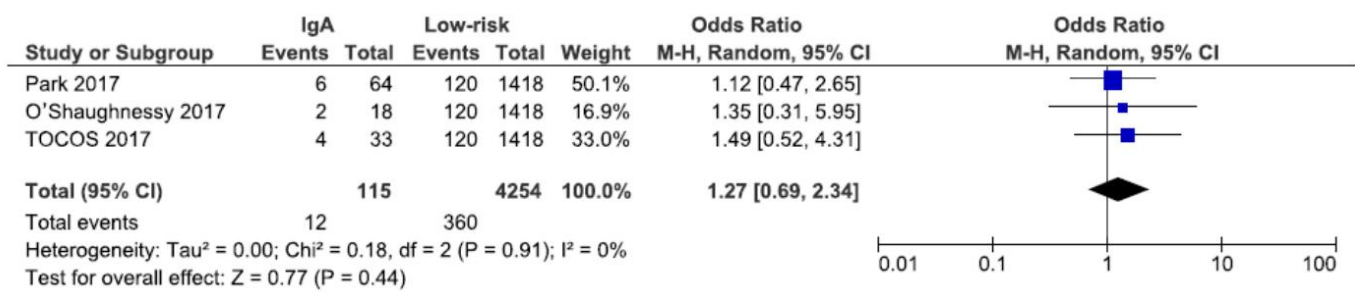

g

\begin{tabular}{|c|c|c|c|c|c|c|c|c|c|c|}
\hline Study or Subgroup & \multicolumn{2}{|c|}{$\lg A$} & \multicolumn{2}{|c|}{ Low-risk } & Weight & $\begin{array}{c}\text { Odds Ratio } \\
\text { M-H, Random, } 95 \% \mathrm{Cl}\end{array}$ & & \multicolumn{2}{|c|}{$\begin{array}{c}\text { Odds Ratio } \\
\text { M-H, Random, } 95 \% \text { Cl }\end{array}$} & \\
\hline Su 2017 & 62 & 116 & 379 & 1418 & $21.1 \%$ & $3.15[2.15,4.62]$ & & & 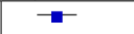 & \\
\hline TOCOS 2017 & 9 & 33 & 379 & 1418 & $16.5 \%$ & $1.03[0.47,2.23]$ & & & & \\
\hline Liu 2014 & 42 & 69 & 379 & 1418 & $19.9 \%$ & $4.26[2.59,7.01]$ & & & $\rightarrow-$ & \\
\hline Oh 2011 & 24 & 52 & 379 & 1418 & $19.2 \%$ & $2.35[1.35,4.10]$ & & & $\rightarrow-$ & \\
\hline Shimizu 2010 & 5 & 29 & 379 & 1418 & $14.2 \%$ & $0.57[0.22,1.51]$ & & & & \\
\hline Waness 2010 & 2 & 12 & 379 & 1418 & $9.0 \%$ & $0.55[0.12,2.51]$ & & & & \\
\hline Total $(95 \% \mathrm{Cl})$ & & 311 & & 8508 & $100.0 \%$ & $1.76[0.98,3.16]$ & & & & \\
\hline Total events & 144 & & 2274 & & & & & & & \\
\hline $\begin{array}{l}\text { Heterogeneity: } \mathrm{Tau}^{2} \\
\text { Test for overall effect }\end{array}$ & $\begin{array}{l}\text { 38; } \mathrm{Chi}^{2} \\
=1.89(\end{array}$ & $\begin{array}{l}=23.8 \\
P=0.0\end{array}$ & $d f=5$ & $(P=0$ & $002) ; 1^{2}$ & & 0.01 & 0.1 & 10 & 100 \\
\hline
\end{tabular}

h

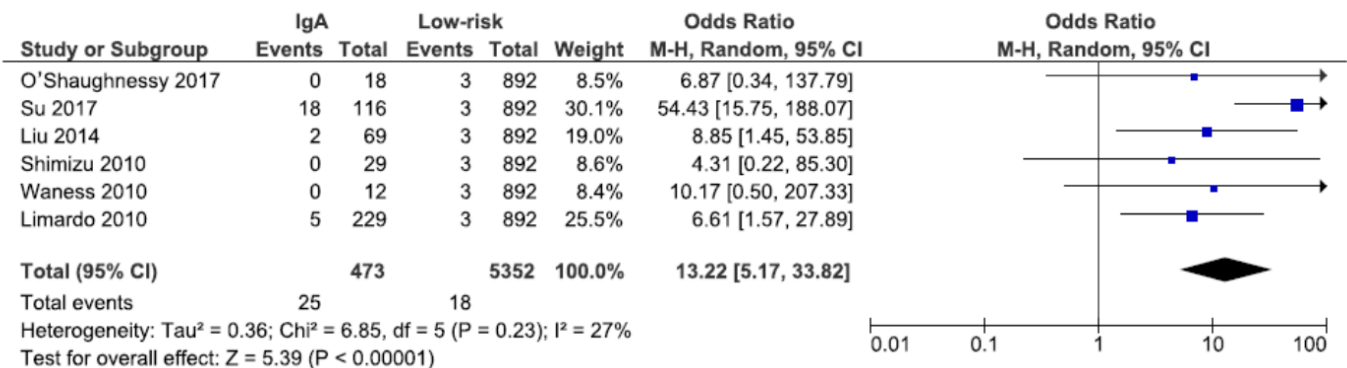

Figure 3. Odds ratio for different pregnancy related outcomes. SGA: small for gestational age. IUGR: intrauterine growth restriction. PE: preeclampsia. CI: confidence intervals.

\section{Discussion}

This systematic review identified 13 cases series and 14 case reports, reporting on pregnancy-related outcomes in IgA nephropathy [27-53]. This is, at the best of our knowledge, the largest number of papers meta-analyzed or narratively discussed on IgA nephropathy in pregnancy, with particular attention to the risk of adverse pregnancy-related outcomes. In this regard, our review may add information to a previous recent systematic review, which included papers published since the start of Medline, and 
was mainly focused on the progression of kidney disease in patients with IgA nephropathy with and without pregnancy [23]. The review, that selected 4 papers with a control group, providing data of 273 patients with IgA nephropathy and of 241 patients with IgA nephropathy who did not become pregnant, supported a lack of disadvantage on the progression of nephropathy for IgA nephropathy patients with well-preserved function that became pregnant.

The main novelty of the present review is to give more insight into the risk of pregnancy-related outcomes, that were already reported as frequent by the previous study, but that are meta-analyzed for the first time with respect to a low-risk population; in this context, limiting the analysis to the studies published since 2000 may allow for a better contextualization in the present panorama (Figure 3).

The pattern of pregnancy-related outcomes is complex and the behavior of the risks may offer some interesting insights into the pathogenesis of pregnancy complications in IgA nephropathy. In fact, the meta-analysis identifies a particularly high risk of PE and PIH in women with IgA nephropathy, with an over ten-fold increase, within wide but consistent confidence intervals (Figure 3). In spite of these high odds ratios, the significantly increased risk of preterm birth was only about triple compared to the low-risk population and the incidence of newborns with low birth weight is only doubled, while no difference was found in the incidence of both caesarean sections and of small for gestational age babies (Figure 3). This finding suggests that the very high risk for hypertensive disorders of pregnancy is not accompanied by a correspondent increase of preterm babies, and of babies whose growth has been severely impaired, as it occurs in the "placental" early forms of preeclampsia, and therefore suggests that the related event occurs later, corresponding with the so-called "late" form of PE [54-58]. Such a form of PE is usually milder, and is often associated with maternal diseases, and indeed some authors distinguish between "maternal" and "placental" preeclampsia, the latter characterized by a primary defect of placentation, and frequently associated with intrauterine growth restriction. Conversely, the maternal form is more often of late onset, frequently, even if not always, less severe, and is associated with a lower risk of newborns that are small for gestational age, a pattern that may be consistent with the observations gathered in the present review [54-59].

With respect to disease progression, the lack of negative effect of pregnancy, at least in populations with relatively preserved kidney function, reported in the previous meta-analysis, is confirmed by our analysis, performed with a different, selection of the papers, and with the chance of adding several recent studies on this issue (Figure 2) [23]. In most of the studies retrieved by both reviews kidney function was normal or well-preserved before pregnancy and, in this context, the progression of the kidney disease does not appear to be affected by pregnancy. However, most studies fail to give information both on women with severe CKD and the duration of the observation time is not always clear. Furthermore, even if relatively long, the median follow-up period of 10 years described by Limardo, and of 4.5 years by Liu, could still be too short to draw conclusions, given the slow progression of early IgA nephropathy $[31,36]$. The interesting new suggestion that progression of kidney disease is limited to the cases who display adverse pregnancy outcomes should be confirmed in further studies [27].

The case reports, as expected, focus on exceptional events, and are probably affected by reporting and publishing biases. Despite these limits, they may underline that positive pregnancy outcomes are possible, even in severe advanced chronic kidney disease [40-53] (Tables 5-7).

The major strength of this study is that it provides an updated systematic review in one of the most common nephropathies observed in young patients all over the world, gathering over 700 pregnancy published in the new millennium, and attempting, for the first time, a risk assessment with regard to the overall population. The integration with case reports, a further novel aspect of this review, underlines that a positive outcome is possible even in women with severe CKD, who are usually excluded from the larger case series.

The main limitations of this review are linked to the heterogeneity of the studies in terms of populations, definitions and outcomes, as well as duration of follow-up. Heterogeneity is a common problem in systematic reviews on CKD pregnancies, which limits the value of the meta-analysis, 
and calls for a common language, a still unmet goal [60-62]. Furthermore, due to lack of data on low-risk populations, we employed the only available one, from a multicenter Italian study; the approach of choosing a reference population for a wider meta-analysis is not new, and was for example employed by Deshpande and coworkers in the pivotal analysis on pregnancy after kidney or after liver transplantation, which plotted the data against the reference USA population $[63,64]$. Such a choice is an obvious compromise, and the lack of matched populations limits the precision of the comparison; however, the differences with the low-risk populations are highly significant, and their pattern is consistent, thus allowing at least posing new hypotheses and indications for future research.

Within these limits, we hope that our findings may be of interest for counseling and for tailoring clinical surveillance for clinicians working in obstetrics and in nephrology.

Our study may also indicate uncovered fields for future research. First of all, more data is needed to better define the risk of adverse pregnancy outcomes, particularly so in case of kidney function decrease. Secondly, given the impossibility to clinically distinguish an increase in proteinuria and hypertension related to the IgA nephropathy from that related to preeclampsia, there is need for precise description of these cases, with stratification for previous hypertension, and univocal definitions of superimposed PE. In this regard, IgA nephropathy may represent a field for the systematic use of old and new biomarkers of PE and kidney disease [63-68].

\section{Conclusions}

Patients with IgA nephropathy and severe CKD should be informed about the paucity of data allowing a precise quantification of the risks. In patients with preserved kidney function, that represent the majority of those reported, the risk of progression of kidney disease is low, and may be limited to the cases who display adverse pregnancy related events. Conversely the risk of developing PE and $\mathrm{PIH}$ is very high compared to the low-risk population (OR $>10)$. The pattern of these hypertensive disorders of pregnancy, associated with a significant but milder increase in preterm delivery but not of small for gestational age babies, suggests the presence of late onset PE, in its "maternal" form, thought to affect fetal growth less severely than 'placental' early PE. This hypothesis, needing confirmation on a larger scale, may prove of interest in interpreting the increase in hypertensive disorders of pregnancy observed in chronic kidney disease.

Author Contributions: G.B.P., G.C. and R.A. contributed to development of research question and review design. I.A.K. and G.B.P. led literature search, data extraction. Interpretation of results was done by G.B.P., I.A.K., R.A. and T.T. Analysis was performed by M.B., E.V. and I.A.K. Writing of the paper involved G.B.P., I.A.K., R.A. and T.T., R.A., F.F., M.G., E.V. and G.C.; B.M.; F.F. and M.G. gathered the control data (low-risk pregnancies) and contributed to interpretation of results and writing. Equivalent participation for the first two and the last three authors.

Conflicts of Interest: The authors declare no conflict of interest.

\section{References}

1. Floege, J.; Amann, K. Primary glomerulonephritides. Lancet 2016, 387, 2036-2048. [CrossRef]

2. Kurokawa, M.; Hisano, S.; Ueda, K. Berger disease/Henoch-Schönlein syndrome. J. Pediatr. 1985, 107, 648-649. [CrossRef]

3. Novak, J.; Renfrow, M.B.; Gharavi, A.G.; Julian, B.A. Pathogenesis of immunoglobulin A nephropathy. Curr. Opin. Nephrol. Hypertens. 2013, 22, 287-294. [CrossRef] [PubMed]

4. Feehally, J.; Barratt, J. The genetics of IgA nephropathy: An overview from Western countries. Kidney Dis. 2015, 1, 33-41. [CrossRef] [PubMed]

5. Zhu, L.; Zhang, H. The genetics of IgA nephropathy: An overview from China. Kidney Dis. 2015, 1, $27-32$. [CrossRef] [PubMed]

6. Scolari, F. Familial IgA nephropathy. J. Nephrol. 1999, 12, 213-219. [PubMed]

7. Lee, H.; Hwang, J.H.; Paik, J.H.; Ryu, H.J.; Kim, D.K.; Chin, H.J.; Oh, Y.K.; Joo, K.W.; Lim, C.S.; Kim, Y.S.; et al. Long-term prognosis of clinically early IgA nephropathy is not always favorable. BMC Nephrol. 2014, 15, 94. [CrossRef] [PubMed] 
8. Moriyama, T.; Tanaka, K.; Iwasaki, C.; Oshima, Y.; Ochi, A.; Kataoka, H.; Itabashi, M.; Takei, T.; Uchida, K.; Nitta, K.; et al. Prognosis in IgA nephropathy: 30-year analysis of 1012 patients at a single center in Japan. PLoS ONE 2014, 9, e91756. [CrossRef] [PubMed]

9. Tomino, Y. Immunopathological predictors of prognosis in IgA nephropathy. Contrib. Nephrol. 2013, 181, 65-74. [PubMed]

10. Kawamura, T.; Joh, K.; Okonogi, H.; Koike, K.; Utsunomiya, Y.; Miyazaki, Y.; Matsushima, M.; Yoshimura, M.; Horikoshi, S.; Suzuki, Y.; et al. Study Group Special IgA Nephropathy. A histologic classification of IgA nephropathy for predicting long-term prognosis: Emphasis on end-stage renal disease. J. Nephrol. 2013, 26, 350-357. [CrossRef] [PubMed]

11. Maixnerova, D.; Reily, C.; Bian, Q.; Neprasova, M.; Novak, J.; Tesar, V. Markers for the progression of IgA nephropathy. J. Nephrol. 2016, 29, 535-541. [CrossRef] [PubMed]

12. Working Group of the International IgA Nephropathy Network and the Renal Pathology Society; Roberts, I.S.; Cook, H.T.; Troyanov, S.; Alpers, C.E.; Amore, A.; Barratt, J.; Berthoux, F.; Bonsib, S.; Bruijn, J.A.; et al. The Oxford classification of IgA nephropathy: Pathology definitions, correlations, and reproducibility. Kidney Int. 2009, 76, 546-556. [CrossRef] [PubMed]

13. Reich, H.N.; Troyanov, S.; Scholey, J.W.; Cattran, D.C. Toronto Glomerulonephritis Registry. Remission of proteinuria improves prognosis in IgA nephropathy. J. Am. Soc. Nephrol. 2007, 18, 3177-3183. [CrossRef] [PubMed]

14. Usui, J.; Yamagata, K.; Kai, H.; Outeki, T.; Yamamoto, S.; Muro, K.; Hirayama, A.; Yoh, K.; Tomida, C.; Hirayama, K.; et al. Heterogeneity of prognosis in adult IgA nephropathy, especially with mild proteinuria or mild histological features. Intern. Med. 2001, 40, 697-702. [CrossRef] [PubMed]

15. Coppo, R.; Lofaro, D.; Camilla, R.R.; Bellur, S.; Cattran, D.; Cook, H.T.; Roberts, I.S.; Peruzzi, L.; Amore, A.; Emma, F.; et al. Risk factors for progression in children and young adults with IgA nephropathy: An analysis of 261 cases from the VALIGA European cohort. Pediatr. Nephrol. 2017, 32, 139-150. [CrossRef] [PubMed]

16. Pesce, F.; Diciolla, M.; Binetti, G.; Naso, D.; Ostuni, V.C.; Di Noia, T.; Vågane, A.M.; Bjørneklett, R.; Suzuki, H.; Tomino, Y.; et al. Clinical decision support system for end-stage kidney disease risk estimation in $\operatorname{IgA}$ nephropathy patients. Nephrol. Dial. Transplant. 2016, 31, 80-86. [CrossRef] [PubMed]

17. Sarcina, C.; Tinelli, C.; Ferrario, F.; Pani, A.; De Silvestri, A.; Scaini, P.; Del Vecchio, L.; Alberghini, E.; Buzzi, L.; Baragetti, I.; et al. Changes in proteinuria and side effects of corticosteroids alone or in combination with azathioprine at different stages of IgA nephropathy. Clin. J. Am. Soc. Nephrol. 2016, 11, 973-981. [CrossRef] [PubMed]

18. Coppo, R. Is a legacy effect possible in IgA nephropathy? Nephrol. Dial. Transplant. 2013, 28, 1657-1662. [CrossRef] [PubMed]

19. Piccoli, G.B.; Cabiddu, G.; Attini, R.; Vigotti, F.N.; Maxia, S.; Lepori, N.; Tuveri, M.; Massidda, M.; Marchi, C.; Mura, S.; et al. Risk of adverse pregnancy outcomes in women with CKD. J. Am. Soc. Nephrol. 2015, 26, 2011-2022. [CrossRef] [PubMed]

20. Bramham, K.; Lightstone, L. Pre-pregnancy counseling for women with chronic kidney disease. J. Nephrol. 2012, 25, 450-459. [CrossRef] [PubMed]

21. Tong, A.; Brown, M.A.; Winkelmayer, W.C.; Craig, J.C.; Jesudason, S. Perspectives on pregnancy in women with CKD: A semistructured interview Study. Am. J. Kidney Dis. 2015, 66, 951-961. [CrossRef] [PubMed]

22. Piccoli, G.B.; Cabiddu, G.; Attini, R.; Vigotti, F.; Fassio, F.; Rolfo, A.; Giuffrida, D.; Pani, A.; Gaglioti, P.; Todros, T.; et al. Pregnancy in chronic kidney disease: Questions and answers in a changing panorama. Best Pract. Res. Clin. Obstet. Gynaecol. 2015, 29, 625-642. [CrossRef] [PubMed]

23. Liu, Y.; Ma, X.; Zheng, J.; Liu, X.; Yan, T. A systematic review and meta-analysis of kidney and pregnancy outcomes in IgA nephropathy. Am. J. Nephrol. 2016, 44, 187-193. [CrossRef] [PubMed]

24. Johnson, D.W.; Atai, E.; Chan, M.; Phoon, R.K.; Scott, C.; Toussaint, N.D.; Turner, G.L.; Usherwood, T.; Wiggins, K.J.; KHA-CARI. KHA-CARI guideline: Early chronic kidney disease: Detection, prevention and management. Nephrology 2013, 18, 340-350. [CrossRef] [PubMed]

25. Cabiddu, G.; Castellino, S.; Gernone, G.; Santoro, D.; Moroni, G.; Giannattasio, M.; Gregorini, G.; Giacchino, F.; Attini, R.; Loi, V.; et al. A best practice position statement on pregnancy in chronic kidney disease: The Italian Study Group on Kidney and Pregnancy. J. Nephrol. 2016, 29, 277-303. [CrossRef] [PubMed]

26. Blom, K.; Odutayo, A.; Bramham, K.; Hladunewich, M.A. Pregnancy and glomerular disease: A systematic review of the literature with management guidelines. Clin. J. Am. Soc. Nephrol. 2017. [CrossRef] [PubMed] 
27. Park, S.; Yoo, K.D.; Park, J.S.; Hong, J.S.; Baek, S.; Park, S.K.; Chin, H.J.; Na, K.Y.; Choi, Y.; Kim, D.K.; et al. Pregnancy in women with immunoglobulin A nephropathy: Are obstetrical complications associated with renal prognosis? Nephrol. Dial. Transplant. 2017, 33, 459-465. [CrossRef] [PubMed]

28. O'Shaughnessy, M.M.; Jobson, M.A.; Sims, K.; Liberty, A.L.; Nachman, P.H.; Pendergraft, W.F. pregnancy outcomes in patients with glomerular disease attending a single Academic Center in North Carolina. Am. J. Nephrol. 2017, 45, 442-451. [CrossRef] [PubMed]

29. Su, X.; Lv, J.; Liu, Y.; Wang, J.; Ma, X.; Shi, S.; Liu, L.; Zhang, H. Pregnancy and kidney outcomes in patients with IgA nephropathy: A cohort study. Am. J. Kidney Dis. 2017. [CrossRef] [PubMed]

30. Piccoli, G.B.; Attini, R.; Cabiddu, G.; Kooij, I.; Fassio, F.; Gerbino, M.; Maxia, S.; Biolcati, M.; Versino, E.; Todros, T.; et al. Maternal-foetal outcomes in pregnant women with glomerulonephritides. Are all glomerulonephritides alike in pregnancy? J. Autoimmun. 2017, 79, 91-98. [CrossRef] [PubMed]

31. Liu, Y.; Ma, X.; Lv, J.; Shi, S.; Liu, L.; Chen, Y.; Zhang, H. Risk factors for pregnancy outcomes in patients with IgA nephropathy: A matched cohort study. Am. J. Kidney Dis. 2014, 64, 730-736. [CrossRef] [PubMed]

32. Oh, H.J.; Han, S.H.; Yoo, D.E.; Kim, S.J.; Park, J.T.; Kim, J.K.; Yoo, T.H.; Kang, S.W.; Choi, K.H. Reduced pre-pregnancy proteinuria is associated with improving postnatal maternal renal outcomes in IgA nephropathy women. Clin. Nephrol. 2011, 76, 447-454. [CrossRef] [PubMed]

33. Suetsugu, Y.; Tokudome, G.; Sugano, N.; Yoshizawa, T.; Endo, S.; Hara, Y.; Takane, K.; Kuriyama, S.; Hosoya, T. Study on the predictors for superimposed preeclampsia in patients with IgA nephropathy. Nihon Jinzo Gakkai Shi 2011, 53, 1139-1149. [PubMed]

34. Shimizu, A.; Takei, T.; Moriyama, T.; Itabashi, M.; Uchida, K.; Nitta, K. Effect of kidney disease stage on pregnancy and delivery outcomes among patients with immunoglobulin A nephropathy. Am. J. Nephrol. 2010, 32, 456-461. [CrossRef] [PubMed]

35. Waness, A.; Al Sayyari, A.; Salih, S.B.; Al Shohaib, S. Increased risk of hypertension, proteinuria and preeclampsia in pregnant Saudi females with IgA nephropathy. Hypertens. Pregnancy 2010, 29, 385-389. [CrossRef] [PubMed]

36. Limardo, M.; Imbasciati, E.; Ravani, P.; Surian, M.; Torres, D.; Gregorini, G.; Magistroni, R.; Casellato, D.; Gammaro, L.; Pozzi, C.; et al. Pregnancy and progression of IgA nephropathy: Results of an Italian multicenter study. Am. J. Kidney Dis. 2010, 56, 506-512. [CrossRef] [PubMed]

37. Donggyu, J.; Jisun, W.; Sekyoung, C.; Jaeun, S.; Inyang, P.; Jongchul, S. IgA nephropathy in pregnancy. J. Matern.-Fetal Neonatal Med. 2010, 23 (Suppl. 1), 205.

38. Ronkainen, J.; Ala-Houhala, M.; Autio-Harmainen, H.; Jahnukainen, T.; Koskimies, O.; Merenmies, J.; Mustonen, J.; Örmälä, T.; Turtinen, J.; Nuutinen, M.; et al. Long-term outcome 19 years after childhood IgA nephritis: A retrospective cohort study. Pediatr. Nephrol. 2006, 21, 1266-1273. [CrossRef] [PubMed]

39. Ronkainen, J.; Nuutinen, M.; Koskimies, O. The adult kidney 24 years after childhood Henoch-Schonlein purpura: A retrospective cohort study. Lancet 2002, 360, 666-670. [CrossRef]

40. Kaul, A.; Pradhan, M.; Bhaduaria, D.; Prasad, N.; Gupta, A.; Sharma, R. Nephrotic syndrome in pregnancy maternal and foetal outcome. Nephrology 2016, 21 (Suppl. 2), 237-238.

41. Lim, D.; Smith, M.P. Lost to follow up. J. Gen. Intern. Med. 2016, 31 (Suppl. 1), S665-S666.

42. Sun, L.X.; Ye, W.L.; Wen, Y.B.; Li, X.M. Postpartum atypical hemolytic uremic syndrome: An unusual and severe complication associated with IgA nephropathy. Chin. Med. Sci. J. 2015, 30, 189-192. [CrossRef]

43. Nagai, K.; Kishi, J.; Morizumi, S.; Minakuchi, J.; Bando, Y.; Nishioka, Y.; Doi, T. Henoch-Schonlein purpura nephritis occurring postpartum in a patient with anti-PL-7 anti-synthetase syndrome. Mod. Rheumatol. 2015, 27, 910-913. [CrossRef] [PubMed]

44. Liang, K.; Bastacky, S. IGA nephropathy presenting during pregnancy. Am. J. Kidney Dis. 2015, 65, A54.

45. Zand, L.; Williams, A.; Babovic-Vuksanovic, D.; Nwoko, R.; Cornell, L.; Garovic, V. The Case। Renal dysfunction in a pregnant patient with IgA nephropathy. Kidney Int. 2014, 85, 1477-1478. [CrossRef] [PubMed]

46. Cornelis, T.; Spaanderman, M.; Beerenhout, C.; Perschel, F.H.; Verlohren, S.; Schalkwijk, C.G.; van der Sande, F.M.; Kooman, J.P.; Hladunewich, M. Antiangiogenic factors and maternal hemodynamics during intensive hemodialysis in pregnancy. Hemodial. Int. 2013, 17, 639-643. [CrossRef] [PubMed]

47. Hou, S. A woman with GN presenting during pregnancy. Clin. J. Am. Soc. Nephrol. 2013, 8, 1027-1033. [CrossRef] [PubMed] 
48. Giofre, F.; Pugliese, C.; Alati, G.; Messina, A.; Tramontana, D. Three successive pregnancies in a patient with chronic renal disease progressing from chronic renal dysfunction through to institution of dialysis during pregnancy and then on to maintenance dialysis. Nephrol. Dial. Transplant. 2007, 22, 1236-1240. [CrossRef] [PubMed]

49. Tanno, Y.; Yamamoto, H.; Yamamoto, I.; Yaginuma, T.; Mitome, J.; Kawamura, Y.; Miyazaki, Y.; Yokoyama, K.; Utsunomiya, Y.; Yamaguchi, Y.; et al. Recurrence of Henoch-Schönlein purpura nephritis superimposed on severe pre-eclampsia in a kidney transplant patient. Clin. Transplant. 2007, 21 (Suppl. 18), 36-39. [CrossRef]

50. Barquero-Romero, J.; Chaves-Alvarez, A.J.; Catalina-Fernandez, I.; Lopez-Cortezon, C. Schonlein-Henoch purpure in a pregnant patient. Med. Clin. 2006, 127, 276-277. [CrossRef]

51. Koizumi, M.; Hagino, D.; Fukuyama, C.; Abe, K.; Inoue, K.; Arai, Y.; Takechi, K. Schonlein-Henoch purpura during pregnancy: Case report and review of the literature. J. Obstet. Gynaecol. Res. 2004, 30, 37-41. [CrossRef] [PubMed]

52. Cusi, D.; Taglietti, M.V.; Liccardo, A. Pregnancy during nephropathy. Giornale Italiano di Nefrologia 2003, 20, 516-524. [PubMed]

53. Amir, A.R.; Sheikh, S.S. ANCA-associated crescentic IgA glomerulonephritis in pregnancy. J. Nephrol. 2002, 15, 716-719. [PubMed]

54. Lisonkova, S.; Joseph, K.S. Incidence of preeclampsia: Risk factors and outcomes associated with earlyversus late-onset disease. Am. J. Obstet. Gynecol. 2013, 209, 544.e1-544.e12. [CrossRef] [PubMed]

55. Lisonkova, S.; Sabr, Y.; Mayer, C.; Young, C.; Skoll, A.; Joseph, K.S. Maternal morbidity associated with early-onset and late-onset preeclampsia. Obstet. Gynecol. 2014, 124, 771-781. [CrossRef] [PubMed]

56. Ni, Y.; Cheng, W. Comparison of indications of pregnancy termination and prognosis of mothers and neonates in early- and late-onset preeclampsia. Hypertens. Pregnancy 2016, 35, 315-322. [CrossRef] [PubMed]

57. Phipps, E.; Prasanna, D.; Brima, W.; Jim, B. Preeclampsia: Updates in pathogenesis, definitions, and guidelines. Clin. J. Am. Soc. Nephrol. 2016, 11, 1102-1113. [CrossRef] [PubMed]

58. Piccoli, G.B.; Cabiddu, G.; Castellino, S.; Gernone, G.; Santoro, D.; Moroni, G.; Spotti, D.; Giacchino, F.; Attini, R.; Limardo, M.; et al. A best practice position statement on the role of the nephrologist in the prevention and follow-up of preeclampsia: The Italian study group on kidney and pregnancy. J. Nephrol. 2017, 30, 307-317. [CrossRef] [PubMed]

59. Masuyama, H.; Nobumoto, E.; Okimoto, N.; Inoue, S.; Segawa, T.; Hiramatsu, Y. Superimposed preeclampsia in women with chronic kidney disease. Gynecol. Obstet. Investig. 2012, 74, 274-281. [CrossRef] [PubMed]

60. Nevis, I.F.; Reitsma, A.; Dominic, A.; McDonald, S.; Thabane, L.; Akl, E.A.; Hladunewich, M.; Akbari, A.; Joseph, G.; Sia, W.; et al. Pregnancy outcomes in women with chronic kidney disease: A systematic review. Clin. J. Am. Soc. Nephrol. 2011, 6, 2587-2598. [CrossRef] [PubMed]

61. Piccoli, G.B.; Conijn, A.; Attini, R.; Biolcati, M.; Bossotti, C.; Consiglio, V.; Deagostini, M.C.; Todros, T. Pregnancy in chronic kidney disease: Need for a common language. J. Nephrol. 2011, 24, 282-299. [CrossRef] [PubMed]

62. Zhang, J.J.; Ma, X.X.; Hao, L.; Liu, L.J.; Lv, J.C.; Zhang, H. A systematic review and meta-analysis of outcomes of pregnancy in CKD and CKD outcomes in pregnancy. Clin. J. Am. Soc. Nephrol. 2015, 10, 1964-1978. [CrossRef] [PubMed]

63. Deshpande, N.A.; James, N.T.; Kucirka, L.M.; Boyarsky, B.J.; Garonzik-Wang, J.M.; Montgomery, R.A.; Segev, D.L. Pregnancy outcomes in kidney transplant recipients: A systematic review and meta-analysis. Am. J. Transplant. 2011, 11, 2388-2404. [CrossRef] [PubMed]

64. Rolfo, A.; Attini, R.; Nuzzo, A.M.; Piazzese, A.; Parisi, S.; Ferraresi, M.; Todros, T.; Piccoli, G.B. Chronic kidney disease may be differentially diagnosed from preeclampsia by serum biomarkers. Kidney Int. 2013, 83, 177-181. [CrossRef] [PubMed]

65. Piccoli, G.B.; Gaglioti, P.; Attini, R.; Parisi, S.; Bossotti, C.; Olearo, E.; Oberto, M.; Ferraresi, M.; Rolfo, A.; Versino, E.; et al. Pre-eclampsia or chronic kidney disease? The flow hypothesis. Nephrol. Dial. Transplant. 2013, 28, 1199-1206. [CrossRef] [PubMed]

66. Perni, U.; Sison, C.; Sharma, V.; Helseth, G.; Hawfield, A.; Suthanthiran, M.; August, P. Angiogenic factors in superimposed preeclampsia: A longitudinal study of women with chronic hypertension during pregnancy. Hypertension 2012, 59, 740-746. [CrossRef] [PubMed] 
67. Masuyama, H.; Suwaki, N.; Nakatsukasa, H.; Masumoto, A.; Tateishi, Y.; Hiramatrsu, Y. Circulating angiogenic factors in preeclampsia, gestational proteinuria, and preeclampsia superimposed on chronic glomerulonephritis. Am. J. Obstet. Gynecol. 2006, 194, 551-556. [CrossRef] [PubMed]

68. Bramham, K.; Seed, P.T.; Lightstone, L.; Nelson-Piercy, C.; Gill, C.; Webster, P.; Poston, L.; Chappell, L.C. Diagnostic and predictive biomarkers for pre-eclampsia in patients with established hypertension and chronic kidney disease. Kidney Int. 2016, 89, 874-885. [CrossRef] [PubMed]

(C) 2018 by the authors. Licensee MDPI, Basel, Switzerland. This article is an open access article distributed under the terms and conditions of the Creative Commons Attribution (CC BY) license (http:/ / creativecommons.org/licenses/by/4.0/). 